\title{
Profile stiffness and global confinement
}

\author{
X Garbet ${ }^{1}$, P Mantica ${ }^{2}$, F Ryter ${ }^{3}$, G Cordey ${ }^{4}$, F Imbeaux ${ }^{1}$, C Sozzi ${ }^{2}$, \\ A Manini ${ }^{3}, \mathbf{E}$ Asp $^{1}$, V Parail $^{4}, \mathbf{R}^{3}$ Wolf $^{5}$ and the JET EFDA Contributors \\ ${ }^{1}$ Association EURATOM -CEA, CEA Cadarache, 13108 St Paul-Lez-Durance, France \\ 2 Istituto di Fisica del Plasma CNR-EURATOM, via Cozzi 53, 20125 Milano, Italy \\ ${ }^{3}$ MPI für Plasmaphysik, EURATOM-Assoziation, D-8046 Garching bei München, Germany \\ ${ }^{4}$ EURATOM/UKAEA, Culham Science Centre, Abingdon OX14 3DB, UK \\ ${ }^{5}$ Institut für Plasmaphysik, Association EURATOM/FZJ, D-52425 Jülich, Germany
}

Received 5 February 2004

Published 12 July 2004

Online at stacks.iop.org/PPCF/46/1351

doi:10.1088/0741-3335/46/9/002

\begin{abstract}
This paper analyses the properties of a critical gradient transport model based on a few assumptions: electrostatic gyroBohm scaling law, existence of an instability threshold and finite background transport below the threshold. A quantitative criterion of stiffness is proposed, which provides the means for a quantitative assessment and inter-machine comparison. It is also shown that this transport model is compatible with a two-term scaling law of global confinement, as proposed recently by the International Tokamak Physics Activity-Confinement Data Base and Modelling Topical Group. This model has also been applied to analyse a variety of experiments mostly using electron heat modulation on JET, ASDEX-Upgrade, TORE SUPRA and FTU. The thresholds are found to be in the expected domain for micro-instabilities in tokamaks. However, the stiffness factor is found to cover a broad range of variation.
\end{abstract}

(Some figures in this article are in colour only in the electronic version)

\section{Introduction}

The question of profile stiffness in tokamak plasmas has been debated for years [1-7]. Up to now, and in spite of a wealth of results, this debate has not lead to a clear conclusion. This suggests that some conditions have to be fulfilled to observe stiff profiles. Also a new element was introduced in the discussion, which comes from a recent result of the Confinement Data Base and Modelling (CDBM) Topical Group in the frame of the International Tokamak Physics Activity (ITPA). It was found that the energy content is fairly well described by a two term scaling law that separates the contributions of the bulk and the pedestal in H-mode plasmas [8], following an initial proposal to separate core confinement from an edge pedestal constrained by 
MHD stability [9]. These two contributions exhibit different parametric dependences, whereas an assumption of strong stiffness would lead to similar scaling laws.

Part of the disagreement between various studies comes from an ambiguity in the definition of stiffness. Stiff profiles are commonly defined as marginally stable profiles, i.e. profiles whose gradients are very close to the instability threshold everywhere. We will call this situation strong stiffness. A less stringent characterization of strong stiffness is to define a number $\chi_{\mathrm{s}}$, the stiffness factor, which quantifies the ratio between the diffusivity and the difference between the logarithmic gradient of the temperature and its critical value $\kappa_{\mathrm{c}}$, using an appropriate normalization. Strong stiffness corresponds to a large value of $\chi_{s}$. It is also often forgotten that the diffusivity stays finite below the instability threshold. This property is obviously true for ions since the diffusivity cannot be lower than the neoclassical value which is not negligible. The neoclassical diffusivity of electrons is very small. However, it is plausible that a finite turbulent diffusivity exists below the threshold, for instance due to some residual small-scale turbulence or to turbulence propagation. A consequence of finite diffusivity below the instability threshold is the existence of a core region where profiles are subcritical, thus non-stiff. Another feature is the role of boundary conditions. If the edge temperature is low, the temperature logarithmic gradient is above the critical value. Hence a cold edge cannot be strongly stiff. These simple considerations lead to a separation of the plasma into three regions: the core and edge regions, which are not stiff, and a region in between, where the temperature profile is close to marginality. It is quite important to describe these three regions properly. There already exist many transport models that involve an instability threshold and do reproduce these features. One may quote RLW [10], Weiland [11], IFS-PPPL [12], GLF23 [13], Multi-Mode (MMM) [14] and OHE [15] models. The concept of diffusivity with two contributions, a large and a small one, also underlies the mixed Bohm-gyroBohm model [16]. The existence of three regions in the plasma was mentioned in [12]. Stiffness is usually assessed by predicting profiles using one or several models and comparing them with measurements. This has been done extensively during the recent years $[4,7,17,18]$.

The aim of this paper is to address the question of stiffness with a critical gradient transport model that is less simplistic than the concept of 'strong stiffness', but easier to handle than first principles transport models. This critical gradient model is based on an electrostatic gyroBohm scaling hypothesis, and is characterized by three scalar parameters per species: a threshold $\kappa_{\mathrm{c}}$, a stiffness factor $\chi_{\mathrm{s}}$ and a background diffusivity $\chi_{0}$. This set of parameters can be identified by analysing experiments where the heating source is modulated, as already shown by several groups [19-21]. Both steady and modulated profiles provide some information in this case. In particular, the threshold can be determined with accuracy by using the change of slope that is observed on the radial profiles of amplitude and phase of modulated temperature. The drawback is obviously that this simplified model does not cover all the physics known from first principles turbulence simulations. However, it offers many advantages. First, an interpretative analysis can be done in an efficient way, i.e. the parameters of this model can be directly identified from experimental results and compared in different plasma conditions or machines. Second, this transport model has analytical (or semi-analytical) solutions so that some exact results can be obtained and tested. In particular, quantitative conditions for getting stiff profiles can be derived. Finally, it provides an easy access to the scaling law of global confinement. It is stressed here that the objective of this paper is not to propose a new transport model, but rather to propose an effective tool to analyse and compare experiments against theory. The main goal is to determine ranges of variation of the stiffness number $\chi_{\mathrm{s}}$ and threshold $\kappa_{\mathrm{c}}$ from modulation experiments, a two-term global scaling law and theory.

This paper is organized as follows. The transport model is described in section 2. Conditions for stiffness are given in section 3, while the predicted energy content is assessed 
in section 4. Finally, a comparison with experimental results based on existing modulation experiments and on the ITPA-CDBM two-term scaling law is done in section 5. A summary and conclusion follows.

\section{A minimal transport model}

The aim of this section is to derive a simple transport model that preserves some basic properties of turbulent transport. The main hypothesis is a turbulent transport characterized by an electrostatic gyroBohm scaling law, switched on above a threshold $-R \partial_{r} T / T=\kappa_{\mathrm{c}}$ [22] ( $T$ is the temperature and $R$ the major radius). Here $r$ is a label of the flux surface having the dimension of length. The assumption of gyroBohm scaling relies on several recent turbulence simulations in the limit of small values of the normalized gyroradius $\rho^{*}$ [23-26]. However, one has to bear in mind that a substantial departure from gyroBohm scaling is found when the diamagnetic $E \times B$ velocity shear rate is large [23] or when turbulence spreading takes place [26]. Below the threshold, the diffusivity is finite and is produced by collisions for ions, or by some background of turbulence for electrons. To simplify the calculation, the scaling law of this background diffusivity is supposed to be also gyroBohm (this means that the dependence on temperature is wrong when the background diffusivity is neoclassical). With these assumptions, the thermal diffusivity is of the form

$$
\chi_{T}=\chi_{\mathrm{s}} q^{v} \frac{T}{e B} \frac{\rho_{\mathrm{s}}}{R}\left(\frac{-R \partial_{r} T}{T}-\kappa_{\mathrm{c}}\right) H\left(\frac{-R \partial_{r} T}{T}-\kappa_{\mathrm{c}}\right)+\chi_{0} q^{v} \frac{T}{e B} \frac{\rho_{\mathrm{s}}}{R},
$$

where $B$ is the modulus of magnetic field, $q$ is the safety factor, $\rho_{\mathrm{s}}=\sqrt{m_{\mathrm{i}} T} / e B$ is the Larmor radius and $H(x)$ is a Heaviside function. The safety factor has been introduced to account for the improvement of confinement with plasma current. It is also consistent with profiles of diffusivity that increase radially. The exponent $v$ is adjusted once for all when comparing various devices. The value $v=\frac{3}{2}$ was chosen in this work, as it seems to be the best compromise, and allows us to recover the scaling of the two-term scaling law with the plasma current (see section 5). Simulations of ion temperature gradient (ITG) driven turbulence [27,28] and recent dedicated experiments [29] provide some support to this choice. The parameter $v$ was actually found to range between 1 and 2 . Still, this choice cannot be considered as definitive. Once the parameter $v$ is chosen, this transport model is characterized by three dimensionless scalars: the background diffusivity $\chi_{0}$, the stiffness factor $\chi_{\mathrm{s}}$ and the threshold $\kappa_{\mathrm{c}}$, to be determined from experiments. These parameters may depend on plasma parameters, such as the ratio of electron temperature to ion temperature $T_{\mathrm{e}} / T_{\mathrm{i}}$, the effective charge number $Z_{\text {eff }}$, the density gradient length and/or the ratio of the magnetic shear to the safety factor $s / q$ depending on the underlying instability. The present analysis is valid if the three parameters $\chi_{0}, \chi_{\mathrm{s}}$ and $\kappa_{\mathrm{c}}$ are radially uniform for a given plasma. Nevertheless, this hypothesis does not prevent the scalars $\chi_{0}, \chi_{\mathrm{s}}$ and $\kappa_{\mathrm{c}}$ to change from one set of experiments to another one, due to their dependence on the plasma parameters. Still, the assumption that the set $\chi_{0}, \chi_{\mathrm{s}}$ and $\kappa_{\mathrm{c}}$ is radially uniform is a limitation. For instance, the threshold is known to vary radially in Tore Supra [3].

For each species, the steady-state temperature is a solution of the heat equation

$$
\Gamma_{T}=-n \chi_{T} \partial_{r} T
$$

where $n$ is the density and $\Gamma_{T}$ is the heat flux. Using the transport model, equation (1), it can be rewritten as (see appendix A)

$$
\tau^{\prime}\left(\frac{\tau^{\prime}}{\tau}-1\right) H\left(\frac{\tau^{\prime}}{\tau}-1\right)+\lambda_{0} \tau^{\prime}=g
$$


Here $\tau$ is a normalized temperature,

$$
\tau=\left(\frac{T}{T_{\mathrm{gB}}}\right)^{5 / 2}
$$

and is a function of the normalized spatial coordinate ( $r=a$ is the edge boundary)

$$
\rho=\frac{5}{2} \kappa_{\mathrm{c}} \frac{a-r}{R} .
$$

A prime indicates a derivative with respect to $\rho$. The parameter

$$
\lambda_{0}=\frac{\chi_{0}}{\kappa_{\mathrm{c}} \chi_{\mathrm{s}}}
$$

characterizes the relative degree of stiffness and is supposed to be smaller than 1 . The function $g(\rho)$ is a heat flux normalized to the edge value (see appendix A). The temperature $T_{\mathrm{gB}}$ is determined by the relation

$$
\Gamma_{T}(a)=\chi_{\mathrm{s}} \kappa_{\mathrm{c}}^{2} q_{\mathrm{a}}^{v} n_{\mathrm{a}} T_{\mathrm{gB}} \frac{T_{\mathrm{gB}}}{e B} \frac{\rho_{\mathrm{s}, \mathrm{gB}}}{R^{2}},
$$

where the flux $\Gamma_{T}(a)$ is the heat flux at the edge, $q_{\mathrm{a}}$ is the edge safety factor, $n_{\mathrm{a}}$ is the edge density and $\rho_{\mathrm{s}, \mathrm{gB}}=\sqrt{m_{\mathrm{i}} T_{\mathrm{gB}}} / e B$ is the Larmor radius calculated with the temperature $T_{\mathrm{gB}}$. The temperature $T_{\mathrm{gB}}$ plays a central role in this work. It exhibits the usual gyroBohm scaling and may be recast in a more convenient way when the geometry is elliptical (see appendix A)

$T_{\mathrm{gB}, \mathrm{keV}}=1.89\left(\frac{1+\kappa^{2}}{2 \kappa}\right)^{-2 / 5} \chi_{\mathrm{s}}^{-2 / 5} \kappa_{\mathrm{c}}^{-4 / 5} M^{-1 / 5} \varepsilon_{\mathrm{a}}^{-2 / 5} q_{\mathrm{a}}^{-2 v / 5} B_{\mathrm{T}}^{4 / 5} n_{\mathrm{a}, 19}^{-2 / 5} P_{\mathrm{MW}}^{2 / 5}$.

Since each species may be characterized by a different set of parameters $\chi_{0}, \chi_{\mathrm{s}}$ and $\kappa_{\mathrm{c}}$, the temperature $T_{\mathrm{gB}}$ can be different for electron and ions. In expression (8), $P_{\mathrm{MW}}$ is the additional power for one species (in MW), $n_{\mathrm{a}, 19}$ is the edge density in units of $10^{19} \mathrm{~m}^{-3}, B_{\mathrm{T}}$ is the toroidal magnetic field (it is assumed that $B \approx B_{\mathrm{T}}$ ), $\varepsilon_{\mathrm{a}}=a / R$ is the inverse aspect ratio and $M$ is the mass number.

The equation (3) can be rephrased in the following way:

(a) above the threshold, $\tau^{\prime}>\tau$, the temperature gradient is a solution of the equation

$$
\tau^{\prime 2}-\tau\left(1-\lambda_{0}\right) \tau^{\prime}-g \tau=0
$$

or equivalently

$$
\tau^{\prime}=\frac{\left(1-\lambda_{0}\right) \tau}{2}+\left[\frac{\left(1-\lambda_{0}\right)^{2} \tau^{2}}{4}+g \tau\right]^{1 / 2},
$$

(b) below the threshold, $\tau^{\prime}<\tau$, the temperature gradient is a solution of the equation

$$
\tau^{\prime}=\frac{g}{\lambda_{0}} \text {. }
$$

In the case where the normalized heat flux is a radially uniform function or an exponential, the solution of the heat equation (3) is analytical (see appendix B). In the general case, the first order equations (10) and (11) are easily solved numerically. The problem will be illustrated for the choice $\lambda_{0}=0.025$ and a normalized flux $g$ of the form

$$
g=\frac{a}{r} \exp \left[-x_{\mathrm{s}}^{2}\left\{\left(\frac{a}{r}\right)^{2}-1\right\}\right] .
$$

The parameter $x_{\mathrm{s}}$ characterizes the heat source localization and is chosen as $x_{\mathrm{s}}=0.3$ throughout this paper. The boundary condition is an edge temperature $T=T_{\mathrm{a}}$ at $r=\mathrm{a}$. For an $\mathrm{H}$-mode, $T_{\mathrm{a}}$ is the height of the pedestal. From a strict mathematical point of view, the degree of stiffness $\lambda_{0}$ 
and the normalized edge temperature $T_{\mathrm{a}} / T_{\mathrm{gB}}$ are the right parameters to characterize stiffness in this model. However, the experimental determination of the background diffusivity $\chi_{0}$ turns out to be quite difficult in practice. Therefore, many results will be displayed versus the stiffness number $\chi_{\mathrm{s}}$.

\section{Conditions for stiffness}

The analysis of equations (10) and (11) shows that the plasma can be divided into three regions:

(1) An edge region where the temperature is low and the gradient is well above the threshold, i.e. $\tau \ll 1$. An approximate solution of equation (11) is

$$
\tau=\left(\tau_{\mathrm{a}}^{1 / 2}+\frac{1}{2} \int_{0}^{\rho} \mathrm{d} \rho g(\rho)\right)^{2} .
$$

As the temperature increases towards the magnetic axis, the logarithmic gradient gets closer to the threshold and the profile gets stiffer. The transition between the (non-stiff) edge and stiff regions is smooth. By convention, it is decided to set the boundary at $T=T_{\mathrm{gB}}$ (i.e. $\tau=1$ ). Thus a non-stiff edge region exists when $T_{\mathrm{a}} \leqslant T_{\mathrm{gB}}$ (i.e. $\tau_{\mathrm{a}} \leqslant 1$ ).

(2) A stiff region where the temperature is high $T \geqslant T_{\mathrm{gB}}(\tau \geqslant 1)$, and the gradient is above the threshold. An approximate solution when $\lambda_{0} \ll 1$ is the well known exponential shape

$$
\tau \approx C \mathrm{e}^{\rho}
$$

Using the heat flux conservation $\Gamma_{T}=-n_{0} \chi_{T} \partial_{r} T$, it is found that for a stiff profile $\partial_{r} T=-\kappa_{\mathrm{c}} T$, the heat diffusivity is given by $\chi_{T}=\Gamma_{T} /\left(n_{0} \kappa_{\mathrm{c}} T\right)$. Thus the heat diffusivity decreases as the temperature increases along the radial profile. This behaviour may appear paradoxical at first sight. It results from the fact that the temperature increases faster than its gradient, as shown by equation (10) (see also figure 1). Hence the temperature gradient length gets closer to the threshold value when approaching the magnetic axis. It is reminded here that the transition between the edge and stiff regions is smooth. It is decided arbitrarily that the boundary, $\rho=\rho_{\mathrm{gB}}$, between the two regions corresponds to $\tau=1$. For a constant heat flux, the logarithmic derivative of the temperature, $-\partial_{r} T / \kappa_{\mathrm{c}} T$, is a unique function of the temperature $T / T_{\mathrm{gB}}$, as indicated by equation (10). For a given class of heat profiles, all these curves are close to each other (see figure 1).

(3) A region where the temperature is high and its logarithmic derivative is below the threshold. In the following, we will refer to this non-stiff region as the core region. The solution of equation (11) is

$$
\tau=\frac{1}{\lambda_{0}} \int^{\rho} \mathrm{d} \rho g
$$

The transition between the stiff and core regions is sharp and occurs at the position where $\tau=\tau^{\prime}=g / \lambda_{0}$. This equation defines a position $\rho=\rho_{\mathrm{cr}}$ and a critical temperature $T_{\mathrm{cr}}=g\left(\rho_{\mathrm{cr}}\right) T_{\mathrm{gB}} / \lambda_{0}$. Explicit expressions of $\rho_{\mathrm{cr}}$ and $\rho_{\mathrm{gB}}$ are given in appendix B. The transition temperature depends essentially on the reference temperature $T_{\mathrm{gB}}$ and the stiffness factor $\lambda_{0}$. It also depends on the normalized heat flux $g(\rho)$ : when the deposition profile is flatter, the stiff region is thinner. A localized deposition profile leads to a flux $g$ that behaves as $a / r$, thus moving the location of the transition towards the axis. This transition corresponds to the point where the turbulence vanishes (note, however, that a turbulence may propagate from unstable to stable regions). The hot core is therefore a quiescent region (or weakly turbulent, depending on the meaning of $\chi_{0}$ ). 


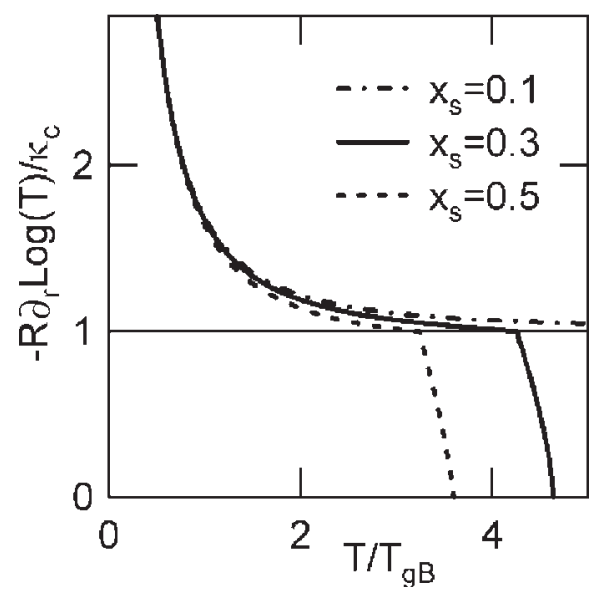

Figure 1. Logarithmic derivative of the temperature versus $T / T_{\mathrm{gB}}$ for $T_{\mathrm{a}} / T_{\mathrm{gB}}=0.5, \lambda_{0}=0.025$ and three values of the heat deposition localization $\left(x_{\mathrm{s}}=0.1,0.3\right.$ and 0.5$)$. The horizontal line is the threshold.

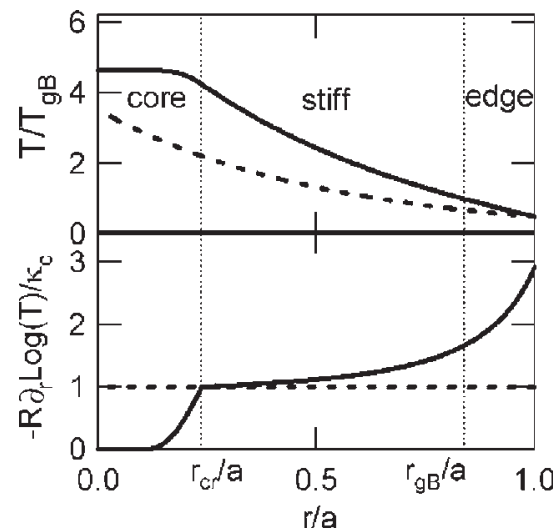

Figure 2. Profiles of the normalized temperature $T / T_{\mathrm{gB}}$ and its logarithmic derivative for $T_{\mathrm{a}} / T_{\mathrm{gB}}=0.5$ and $\lambda_{0}=0.025$. The dashed lines correspond to a stiff profile $T=T_{\mathrm{a}} \mathrm{e}^{\rho}$ (and $\left.-R \partial_{r} T / T=\kappa_{\mathrm{c}}\right)$. The vertical dotted lines are the boundaries of the 'non-stiff' regions $\tau<1$ (edge) and $\tau>g\left(\rho_{\mathrm{cr}}\right) / \lambda_{0}$ (core).

In summary, the temperature profile is stiff in a layer $r_{\mathrm{cr}}<r<r_{\mathrm{gB}}$, where $r_{\mathrm{gB}}$ is the radius such that $\tau=1$, and $r_{\mathrm{cr}} / a=1-\rho_{\mathrm{cr}} / \rho_{\max }$, where $\rho_{\mathrm{cr}}$ is defined above. An example is shown in figure 2 . Figure 3 gives the dependence of these radii on the edge temperature for $\lambda_{0}=0.025$

At this point, three main conclusions may be drawn:

(a) The edge region disappears on increasing the edge temperature, i.e. when $T_{\mathrm{a}} \gg T_{\mathrm{gB}}$. This condition expresses that the edge is stiff when the pedestal is high enough (it comes from the $T^{3 / 2}$ dependence of the diffusivity). We note that $T_{\mathrm{gB}}$ does not depend on the machine size (see equation (8)). So the difference of stiffness observed in various devices has to be explained by means of other considerations. On increasing $T_{\mathrm{a}} / T_{\mathrm{gB}}$, the hot core region broadens, thereby reducing the width of the stiff region. For instance when $T_{\mathrm{a}}=2 T_{\mathrm{gB}}$ and $\lambda_{0}=0.025$, the stiff region only covers half the plasma. 


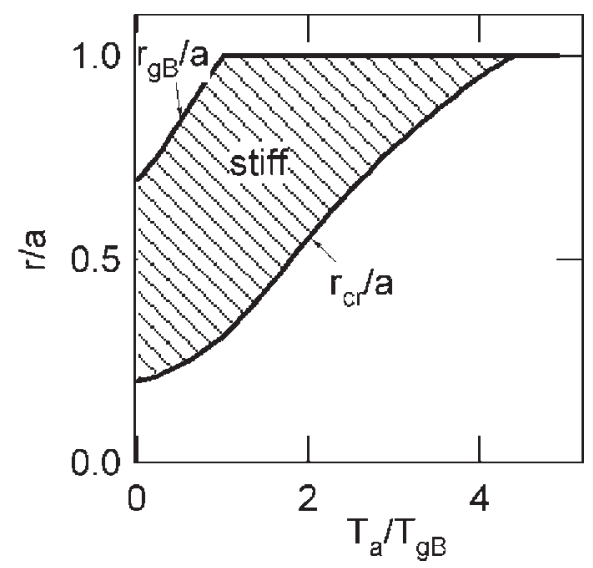

Figure 3. Stiffness layer $\left[r_{\mathrm{cr}} / a, r_{\mathrm{gB}} / a\right]$ for $\lambda_{0}=0.025$ and increasing values of the edge temperature.

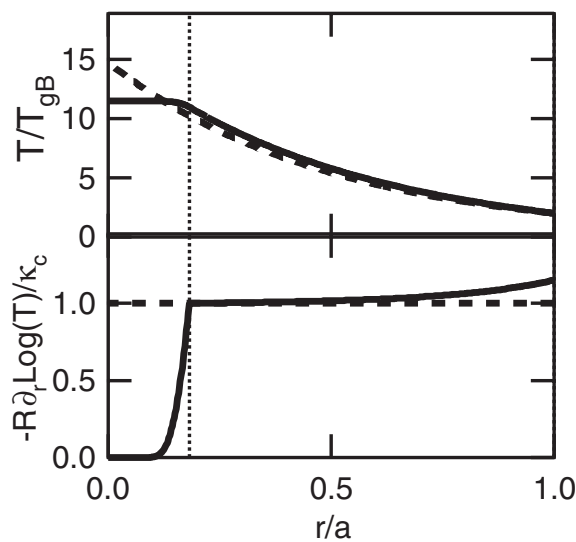

Figure 4. Profiles of the normalized temperature $T / T_{\mathrm{gB}}$ and its logarithmic derivative in a stiff case $T_{\mathrm{a}} / T_{\mathrm{gB}}=2.0$ and $\lambda_{0}=0.001$. Same conventions as in figure 1 .

(b) Stiffness is controlled by the stiffness factor $\chi_{s}$. However, the extension of the core region depends on the relative degree of stiffness $\lambda_{0}=\chi_{0} /\left(\kappa_{\mathrm{c}} \chi_{\mathrm{s}}\right)$. In particular, there is no core region if $\chi_{0}=0$, whatever be the value of $\chi_{\mathrm{s}}$. The transition temperature between the stiff and core regions behaves as $1 / \lambda_{0}$. For instance, for $\lambda_{0}=0.001$, the temperature profile is stiff almost everywhere. An example is shown in figure 4.

(c) A scan of the source radial width indicates that the stiff region is wider when the heat source is more centrally localized (i.e. when the parameter $x_{\mathrm{s}}$ is decreased). This comes from the fact that at a given radius close to the axis the heat flux increases thus maintaining $-\nabla_{r} T / T$ more easily above the threshold. The extension of the non-stiff core region is thus reduced.

In summary, the condition for the plasma to be stiff everywhere is twofold $\tau_{\mathrm{a}}>1$ and $\lambda_{0} \ll 1$, or, equivalently, $T_{\mathrm{a}}>T_{\mathrm{gB}}$ and $\chi_{0} \ll \kappa_{\mathrm{c}} \chi_{\mathrm{s}}$. Peaked deposition profiles enlarge the radial extent of the stiff region. 


\section{Two term scaling law and stiff transport model}

Once the heat equation has been solved for each species, the energy content and confinement time can be calculated. Some simplification is necessary to allow a comparison with a scaling law of energy content. It is assumed here that the model of diffusivity, equation (1) is the same for electrons and ions, with the same threshold $\kappa_{\mathrm{c}}$, degree of stiffness $\lambda_{0}$ and normalized heat flux $g$, but possibly different stiffness factors $\chi_{\mathrm{s}, \mathrm{e}}$ and $\chi_{\mathrm{s}, \mathrm{i}}$. (and, therefore, different background diffusivities to maintain $\lambda_{0}$ at a constant value). Also, the density and the ratio of electron to ion temperatures are supposed to be radially constant. Adding the electron and ion heat equations eliminates the equipartition term. The resulting heat equation is the same as before (i.e. equations (1) and (2)) except that $\chi_{\mathrm{s}}$ is now an effective stiffness factor. Solving in the average temperature $T=\left(T_{\mathrm{e}}+T_{\mathrm{i}}\right) / 2$, this effective stiffness factor appears to be

$$
\chi_{\mathrm{s}, \mathrm{eff}}=\frac{\chi_{\mathrm{s}, \mathrm{i}}+\left(T_{\mathrm{e}} / T_{\mathrm{i}}\right)^{5 / 2} \chi_{\mathrm{s}, \mathrm{e}}}{\left[0.5\left(1+\left(T_{\mathrm{e}} / T_{\mathrm{i}}\right)\right)\right]^{5 / 2}} .
$$

The energy content is then of the form

$$
W=C_{\tau} W_{\mathrm{gB}},
$$

where

$$
C_{\tau}=\frac{1}{\rho_{\max }} \int_{0}^{\rho_{\max }} \mathrm{d} \rho J \tau^{2 / 5}
$$

and $W_{\mathrm{gB}}$ is a gyroBohm energy content that is given in appendix A, equation (A.11). The function $J$ is $J=n V^{\prime} / n_{\mathrm{a}} V_{\mathrm{a}}^{\prime}$. We limit the calculation to the case of an elliptical geometry so that $J=2 r / a=2\left(1-\rho / \rho_{\max }\right)$. It is stressed that $P_{\mathrm{MW}}$ is now the total additional power. The temperature profile and the form factor $C_{\tau}$ are parametrized by the normalized edge temperature $T_{\mathrm{a}} / T_{\mathrm{gB}}$, the stiffness $\lambda_{0}$ and the heat source localization $x_{\mathrm{s}}$. This energy content includes the contribution of the pedestal. Thus, it differs from the core energy content defined by the Confinement Database ITPA group [8]. To avoid any confusion we will call the latter the energy content of the plasma bulk. Hence, the plasma bulk covers the three regions (core, stiff and edge) previously defined. The edge temperature $T_{\mathrm{a}}$ is the height of the pedestal. Following the prescription of the ITPA group, the energy content of the plasma bulk is defined as

$$
W_{E \text { bulk }}=C_{\text {ITPA }} W_{\mathrm{gB}} .
$$

The form factor is now given by the relation

$$
C_{\text {ITPA }}=C_{\tau}-\frac{T_{\mathrm{a}}}{T_{\mathrm{gB}}}=\frac{1}{\rho_{\max }} \int_{0}^{\rho_{\max }} \mathrm{d} \rho J\left(\tau^{2 / 5}-\tau_{\mathrm{a}}^{2 / 5}\right) .
$$

The transport model equation (1) predicts an energy content in the bulk of the form

$$
W_{E \text { bulk }}=0.179 C_{\mathrm{ITPA}} \chi_{\mathrm{s}, \text { eff }}^{-2 / 5} \kappa_{\mathrm{c}}^{-4 / 5} M^{-1 / 5} \kappa^{7 / 5}\left(\frac{1+\kappa^{2}}{2}\right)^{-2 / 5} \varepsilon_{\mathrm{a}}^{8 / 5} R^{3} B_{\mathrm{T}}^{4 / 5} q_{\mathrm{a}}^{-2 v / 5} n_{\mathrm{a}, 19}^{3 / 5} P_{\mathrm{MW}}^{2 / 5}
$$

with the same conventions as the ITPA group. Since the ITPA database is dominated by plasmas with equal ion and electron temperatures, the effective stiffness parameter is $\chi_{\mathrm{s}, \text { eff }}=\chi_{\mathrm{s}, \mathrm{e}+} \chi_{\mathrm{s}, \mathrm{i}}$.

This scaling law is compatible with the ITPA result if the form factor $C_{\text {ITPA }}$ depends weakly on the edge temperature. This is obviously not true in general. It is therefore interesting to analyse the dependence of $C_{\text {ITPA }}$ on the edge temperature. Using the solution found above, 


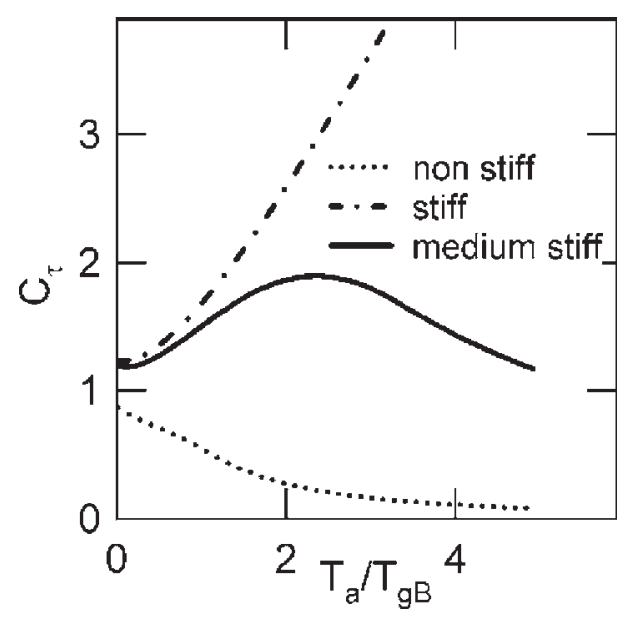

Figure 5. Form factor $C_{\text {ITPA }}$ as function of the edge temperature for three cases: strongly stiff $\left(\lambda_{0}=0.001\right)$, medium stiff $\left(\lambda_{0}=0.05\right)$ and non-stiff $\left(\lambda_{0}=0.9\right)$ cases.

the form factor $C_{\tau}$ is decomposed in the following manner

$$
\begin{aligned}
& C_{\tau}=C_{\tau \text { edge }}+C_{\tau \text { stiff }}+C_{\tau \text { core }} ; \\
& C_{\tau \text { edge }}=\frac{1}{\rho_{\max }} \int_{0}^{\rho_{\mathrm{gB}}} \mathrm{d} \rho J \tau^{2 / 5}, \quad C_{\tau \text { stiff }}=\frac{1}{\rho_{\max }} \int_{\rho_{\mathrm{gB}}}^{\rho_{\mathrm{cr}}} \mathrm{d} \rho J \tau^{2 / 5}, \\
& C_{\tau \text { core }}=\frac{1}{\rho_{\max }} \int_{\rho_{\mathrm{cr}}}^{\rho_{\max }} \mathrm{d} \rho J \tau^{2 / 5} .
\end{aligned}
$$

If the temperature profile is strongly stiff, i.e. if $\tau_{\mathrm{a}} \gg 1$ and $\lambda_{0} \ll 1$, then

$$
C_{\tau \text { edge }}=0, \quad C_{\tau \text { core }}=0 \quad \text { and } \quad C_{\tau \text { stiff }}=2\left(\frac{5}{2 \rho_{\max }}\right)^{2}\left\{\mathrm{e}^{2 \rho_{\max } / 5}-\frac{2 \rho_{\max }}{5}-1\right\} \frac{T_{\mathrm{a}}}{T_{\mathrm{gB}}} .
$$

With the present choice of parameters, this expression reduces to

$$
C_{\text {ITPA }} \approx \frac{1.2 T_{\mathrm{a}}}{T_{\mathrm{gB}}} \text {. }
$$

Therefore, the form factor of a strongly stiff profile is proportional to the edge temperature, as expected. The total energy content is then essentially determined by the pedestal height. An extreme case $\lambda_{0}=0.001$ is shown in figure 5. For a stiff profile the core confinement is not gyroBohm unless the edge temperature $T_{\mathrm{a}}$ follows a gyroBohm scaling law. This is not usually the case. Therefore a strongly stiff profile is incompatible with the result found by the ITPA group.

At this point, a legitimate question is whether a non-stiff transport model that belongs to the class of models of equation (1) fulfils a two-term separation criterion. The answer is negative. This can be understood by using the present transport model in another extreme situation where there is neither a stiff nor an edge region $\left(r_{\mathrm{cr}}=a, \tau_{\mathrm{cr}}=\tau_{\mathrm{a}}\right)$. A simple calculation shows that the form factor varies with edge temperature as

$$
C_{\tau \text { ITPA }, \text { core }} \approx \frac{\rho_{\max }}{5 \lambda_{0}}\left(\frac{T_{\mathrm{a}}}{T_{\mathrm{gB}}}\right)^{-3 / 2} .
$$

This result can be tested by building an artificial case $\lambda_{0}=0.9$ for which the temperature is not stiff for $T_{\mathrm{a}}>T_{\mathrm{gB}}$. As expected from equation (24), it is found that $C_{\text {ITPA }}$ decreases strongly 


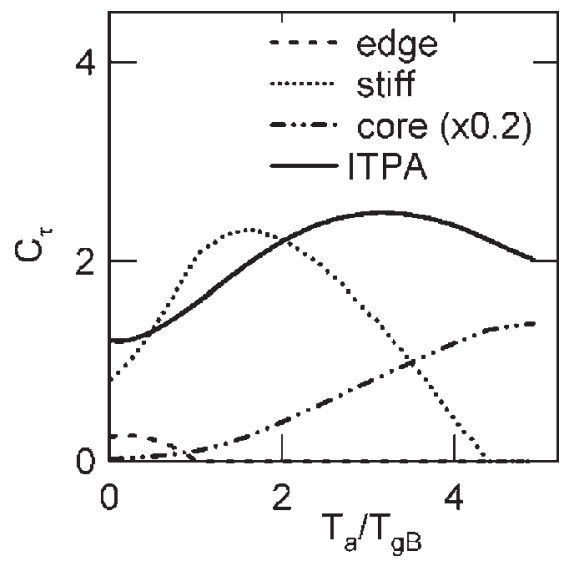

Figure 6. Variations of $C_{\tau \text { edge }}, C_{\tau \text { stiff }}, C_{\tau \text { core }}$ and $C_{\tau \text { ITPA }}$ as functions of the normalized edge temperature for $\lambda_{0}=0.025$.

with the edge temperature (see figure 5). Hence, it does not satisfy the condition for a separation between the pedestal and the bulk. More generally, the separation between the pedestal and bulk is rigorous for a diffusion coefficient that depends on the temperature gradient only (i.e. $\left.\chi_{T} \propto \nabla T^{\alpha}\right)$. For instance, a unique electrostatic gyroBohm model, $\chi_{T} \propto \nabla T^{3 / 2}$, satisfies this requirement. Note that models of this kind (including the gyroBohm version $\chi_{T} \propto \nabla T^{3 / 2}$ ) are not covered by equation (1). This explains why a model based on equation (1) is not compatible with the ITPA findings in the non-stiff limit.

In the general case, the form factor is the sum of edge, stiff and core contributions. As mentioned before, the core contribution increases with the edge temperature whereas the edge contribution decreases. The contribution of the stiff region is non-monotonic. It follows the width of the stiff region. From figure 3, one expects a bell shape. The detailed dependence of these form factors is difficult to assess in general, but can be easily determined numerically. Some asymptotic results are given in appendix C. A 'typical' case $\lambda_{0}=0.025$ is shown in figure 6. It turns out that the trade-off between the edge, stiff and core regions leads to a form factor (as defined by the ITPA-CDBM Group) that is less sensitive to the edge temperature than in the non-stiff or very stiff cases (see the comparison in figure 5). This behaviour occurs in spite of a profile that is stiff over a significant part of the plasma.

\section{Comparison with experiment}

\subsection{Parameters of the transport model}

Several groups have used previously a critical gradient model to analyse heat modulation experiments in ASDEX-Upgrade and JET [19-21]. Models that were used were close but not strictly identical to equation (1) (for instance, the dependence on safety factor was not always included). These shots have been rerun with the same model of equation (1). The aim of this section is not to fully assess the transport model of equation (1) with respect to experiment but rather to determine the range of variation of the parameters of $\chi_{0}, \chi_{\mathrm{s}}$ and $\kappa_{\mathrm{c}}$ deduced from existing experiments.

Electron stiffness is first investigated here, summarizing the results of experiments in ASDEX-Upgrade, JET, FTU and TORE SUPRA. In ASDEX-Upgrade $(R=1.65 \mathrm{~m}$, $a=0.5 \mathrm{~m}$ ), dedicated experiments were performed in L-mode with electron cyclotron resonant 
Table 1. Values of $\chi_{\mathrm{s}, \mathrm{e}}, \chi_{0, \mathrm{e}}, \kappa_{\mathrm{c}, \mathrm{e}}$ and $\lambda_{0, \mathrm{e}}$ in JET, ASDEX-Upgrade, FTU and TORE SUPRA. All pulses are in L-mode except JET \#58148 (H-mode) and ASDEX-Upgrade \#16776-16779 (H-mode).

\begin{tabular}{lllll}
\hline Shot & $\chi_{0, \mathrm{e}}$ & $\chi_{\mathrm{s}, \mathrm{e}}$ & $\kappa_{\mathrm{c}, \mathrm{e}}$ & $\lambda_{0, \mathrm{e}}$ \\
\hline JET 55809 & 0.7 & 4 & 5 & 0.035 \\
JET 55804 & 1.2 & 1.5 & 5 & 0.160 \\
JET 58148 & 0.8 & 3 & 5 & 0.053 \\
JET 53822 & 0.5 & 1.5 & 4.8 & 0.069 \\
JET 55805 & 0.5 & 6 & 5 & 0.017 \\
JET 55802 & 0.5 & 5 & 5 & 0.02 \\
AUG 14793,94 & 0.01 & 0.13 & 3 & 0.026 \\
AUG 17788,89 & 0.01 & 0.13 & 8 & 0.010 \\
AUG 10591 & 0.85 & 0.99 & 6 & 0.143 \\
AUG 12935 & 0.28 & 0.14 & 4 & 0.5 \\
AUG 7806 & 1.79 & 1.4 & 7 & 0.183 \\
AUG 16776,77,79 & $0.05,0.07,0.18$ & $0.2,0.2,0.1$ & $4.5,4.3,4.5$ & $0.056,0.081,0.4$ \\
FTU & 0.7 & 0.5 & 7.5 & 0.187 \\
TS 29214 & 0.05 & 1.42 & 3 & 0.022 \\
\hline
\end{tabular}

heating $(E C R H)$ modulation. The ECRH power was deposited at two radial positions in such a way that the total power was constant. This procedure allowed varying $\nabla T_{\mathrm{e}} / T_{\mathrm{e}}$ over a large range of values while maintaining a constant edge temperature $T_{\mathrm{a}}$. The modulated profiles were modelled as given in equation (24) and led to values of $\chi_{0, \mathrm{e}}, \chi_{\mathrm{s}, \mathrm{e}}$ and $\kappa_{\mathrm{c}, \mathrm{e}}$ [20]. A similar analysis was done in [19] with another set of experiments.

In JET ( $R=3 \mathrm{~m}, a=1 \mathrm{~m})$, experiments using modulated ion cyclotron resonance frequency (ICRF) heating with a steady background of neutral beam injection (NBI) heating have been done in L-mode [21] and H-mode [30]. A mode conversion scheme was used with $18 \%$ of $\mathrm{He}_{3}$ in D plasmas $\left(P_{\mathrm{ICRF}}=3.7 \mathrm{MW}\right)$. Electrons are directly heated when using this ion cyclotron heating scheme, with good localization properties. The position of the mode conversion layer was changed and the NBI power was varied from 2 to $9 \mathrm{MW}$ in order to cover a large range of temperature gradients. The three parameters $\chi_{0, \mathrm{e}}, \chi_{\mathrm{s}, \mathrm{e}}$ and $\kappa_{\mathrm{c}, \mathrm{e}}$ were found by fitting both the steady and modulated profiles [21].

TORE SUPRA data $(R=2.38 \mathrm{~m}, a=0.72 \mathrm{~m})$ comes from modulated ECH experiments in helium plasmas, carried out with one gyrotron providing power deposition at mid-radius. The whole gyrotron power was turned on/off at a frequency of $25 \mathrm{~Hz}$. No other additional heating was applied during the modulation. The three parameters $\chi_{0, \mathrm{e}}, \chi_{\mathrm{s}, \mathrm{e}}$ and $\kappa_{\mathrm{c}, \mathrm{e}}$ have been found by fitting both the steady and modulated profiles.

In FTU $(R=0.96 \mathrm{~m}, a=0.28 \mathrm{~m})$, a series of 9 ECRH heated plasmas were used. The power was not modulated in these experiments, but the heating location was changed. A least squares minimization technique was used to obtain the parameters $\chi_{0, \mathrm{e}}, \chi_{\mathrm{s}, \mathrm{e}}$ and $\kappa_{\mathrm{c}, \mathrm{e}}$.

All these plasmas have been analysed with the same model equation (1), with $v=\frac{3}{2}$. The principal difficulty is the determination of the background diffusivity $\chi_{0, \mathrm{e}}$, given the experimental uncertainties (one reason could be the ad-hoc choice of an electrostatic gyroBohm scaling for the background diffusivity). A sensitivity study of the model was made, as illustrated for shot AUG 14793 (see table 1). This discharge is heated centrally with ECRH only. $\mathrm{A} \pm 10 \%$ power modulation was used to study heat pulse propagation, as described in [20]. A quantitative sensitivity study is done by comparing the time-averaged power balance and heat pulse diffusivities with the respective values yielded by the model. Figure 7 shows the results in which the nominal values of $\chi_{\mathrm{s}}$ and $\kappa_{\mathrm{c}}$ for these shots have been used, as well as values for $\chi_{\mathrm{s}}$ which deviate by $\pm 30 \%$. The sensitivity to $\kappa_{\mathrm{c}}$ is weaker than that to $\chi_{\mathrm{s}}$ in these 

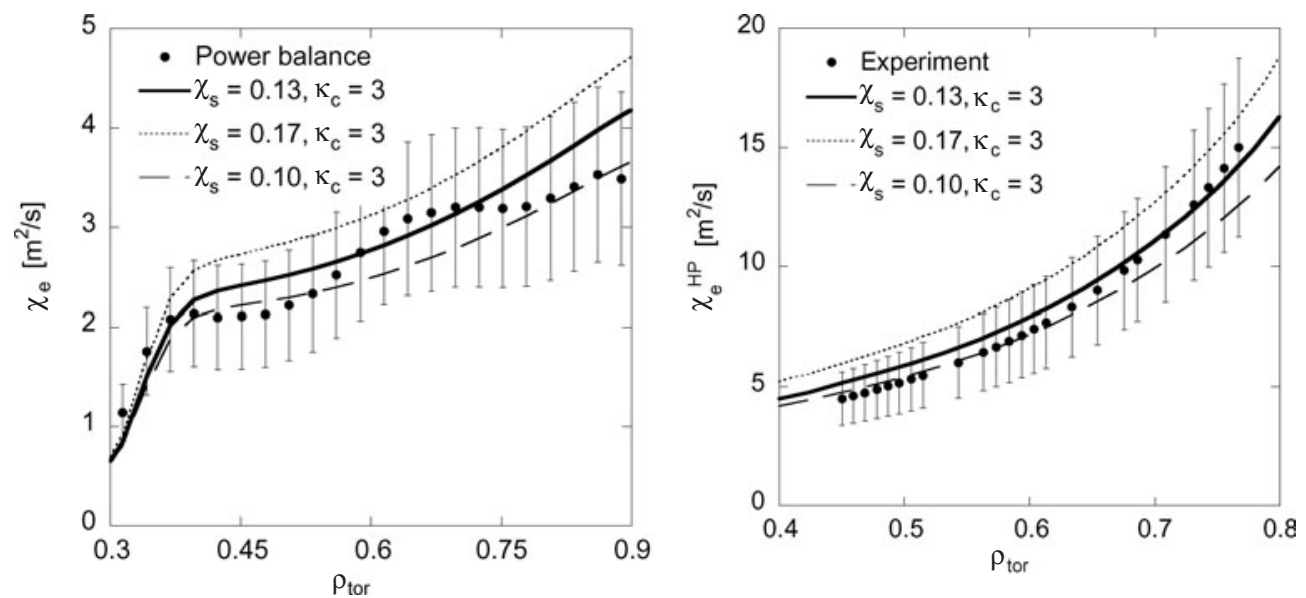

Figure 7. Comparison of experimental power balance and heat pulse diffusivities with the corresponding quantities yielded by the model at three different of $\chi_{\mathrm{s}}$ values (nominal value and values that deviate by $\pm 30 \%$ from nominal).

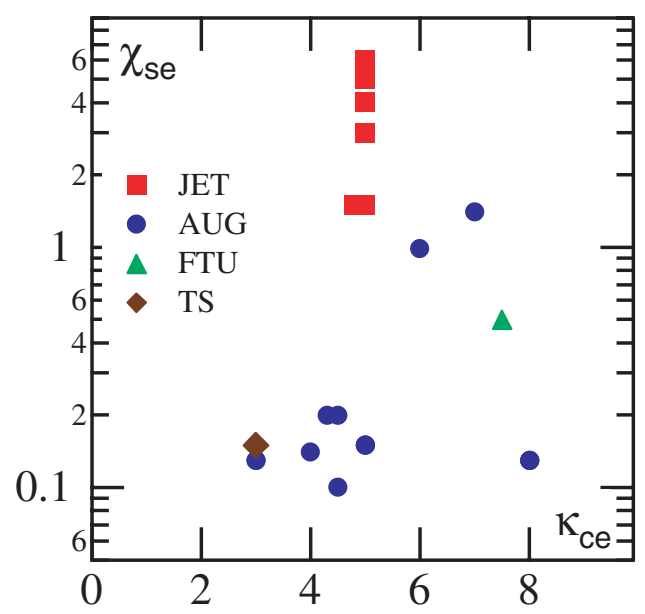

Figure 8. Electron stiffness $\chi_{\mathrm{s}, \mathrm{e}}$ versus threshold $\kappa_{\mathrm{c}, \mathrm{e}}$ deduced from modulation experiments in ASDEX-Upgrade, JET and TORE SUPRA, and from a scan of the position of ECRH heating in FTU.

conditions and it is believed that the method described in [20] yields a more precise value for $\kappa_{\mathrm{c}}$ than can be inferred by adjusting the model. It must be underlined that, of course, the $T_{\mathrm{e}}$ profiles for the cases shown here exhibit only very small deviation compared with the experimental profile. These deviations are difficult to quantify. The quantities $\chi^{\mathrm{PB}}$ and $\chi^{\mathrm{HP}}$ are clearly more sensitive. This study indicates that for such discharges $\chi_{\mathrm{s}}$ and $\kappa_{\mathrm{c}}$ can be determined with a precision of about $\pm 30 \%$. This value is much smaller than the range of variation given in table 1 . This method also indicates that the $q^{3 / 2}$ dependence of the present critical gradient model is adequate for these discharges. Using $q$ or $q^{5 / 2}$ gives, respectively, flatter and steeper slopes for both $\chi^{\mathrm{PB}}$ and $\chi^{\mathrm{HP}}$, which are clearly outside of the error bars.

The result of this multi-machine comparison is shown in table 1 and figure 8 . The thresholds range between 3 and 8 , which are typical values expected for ITG/TEM modes. 

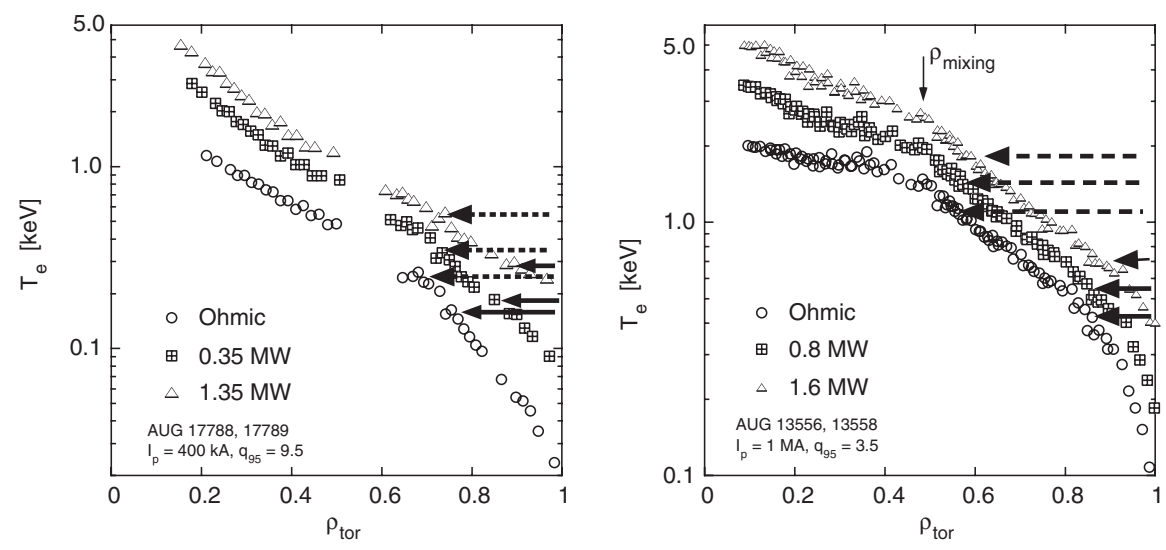

Figure 9. Comparison of $T_{\mathrm{gB}}$ with $T_{\mathrm{e}}$ profiles in ASDEX-Upgrade (L-mode) in Ohmic and ECRH heated plasma. Left panel: $I_{\mathrm{p}}=400 \mathrm{kA}, P_{\mathrm{ECRH}}=0$ (Ohmic), 0.35 and $1.35 \mathrm{MW}$, no sawtooth. Right panel: $I_{\mathrm{p}}=1 \mathrm{MA}, P_{\mathrm{ECRH}}=0$ (Ohmic), 0.8 and $1.6 \mathrm{MW}$, sawtoothing. Dashed horizontal lines indicate $T_{\mathrm{gB}}$ given by equation (8), while horizontal solid lines are the values of $T_{\mathrm{gB}}$ calculated with the values of stiffness parameter and threshold deduced from the ITPA two term scaling law equation (29).

The range of variation of the stiffness parameter $\chi_{\mathrm{s}, \mathrm{e}}$ is found to be much wider, as illustrated by figure 8 . It lies between 0.15 and 6 . The largest values are obtained in JET in plasmas with significant ion heating $\left(\chi_{\mathrm{s}, \mathrm{e}} \sim 3-6\right)$. This variability is surprising, as one would have expected a much smaller range of variation on the basis of simple theoretical considerations. In the JET set of experiments, for example, most plasma parameters are the same, the only variations were performed in the amount of NBI heating and the location of the RF power deposition. One possible explanation for the large variation in the stiffness parameter $\chi_{\mathrm{s}, \mathrm{e}}$ would be a decrease of $\chi_{\mathrm{s}, \mathrm{e}}$ with $T_{\mathrm{e}} / T_{\mathrm{i}}$. However, an analysis of the data indicates this explanation cannot be reconciled with all the points of the database. It seems that the parameter $-R \nabla T_{\mathrm{i}} / T_{\mathrm{i}}$ is better correlated with the variation of $\chi_{\mathrm{s}, \mathrm{e}}$. This suggests a coupling between electron and ion turbulent transport. Further work is needed to confirm this correlation, which requires ion temperature profile measurements (not available for all experiments here).

Ion stiffness is less documented than electron stiffness because the ion temperature is difficult to measure in modulation experiments with the appropriate time resolution. This question has nevertheless been investigated in DIII-D, JET and ASDEX-Upgrade by analysing the steady ion temperature profiles in various regimes [5, 6, 31]. It was found in JET and ASDEX-Upgrade that $\kappa_{\mathrm{c}, \mathrm{i}}$ ranges between 5 and 8 . The scatter is reduced when accounting for the dependence on $T_{\mathrm{e}} / T_{\mathrm{i}}$ and the $E \times B$ velocity shear. For $T_{\mathrm{e}}=T_{\mathrm{i}}$ and low shear rate, the value of the threshold is $\kappa_{\mathrm{c}, \mathrm{i}}=5.3$, which is close to the value found for electrons in JET. The stiffness factor was not obtained in this case, since its value is difficult to obtain with steady-state profiles. In fact, the values of the threshold were obtained by assuming that the profiles are stiff enough to be close to the marginal profiles between $\rho=0.2$ and 0.6. Modulation experiments done at JET are currently under analysis to clarify this issue.

\subsection{Conditions for stiffness}

The transport model equation (1) can be tested in several ways. One is a direct inspection of the temperature profiles. A set of electron temperature profiles coming from ASDEX-Upgrade is shown in figure 9 in Ohmic and ECH L-mode plasmas at $I_{\mathrm{p}}=400 \mathrm{kA}$ and $I_{\mathrm{p}}=1 \mathrm{MA}$ [20]. 


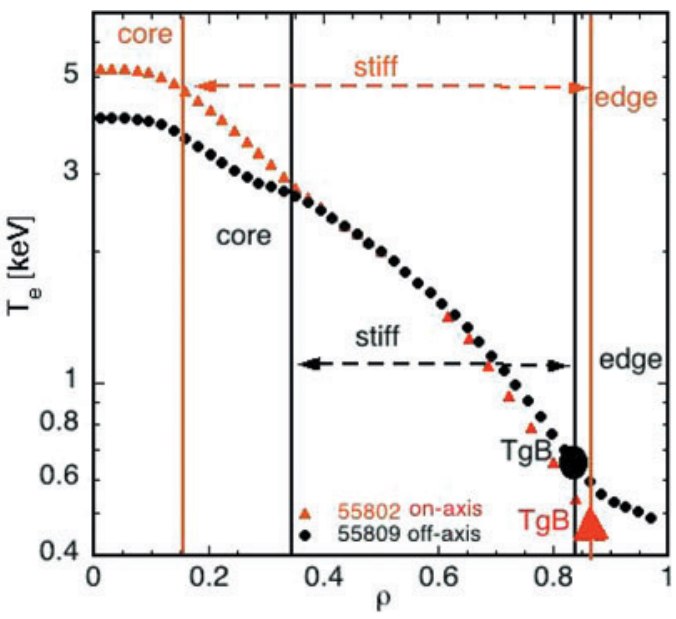

Figure 10. Experimental ECE $T_{\mathrm{e}}$ profiles for two JET discharges with on-axis and off-axis ICRH heating. 55802 (red/grey): $3.6 \mathrm{MW} \mathrm{ICRH}$ on-axis, 3.2 MW NBI; 55809 (black): $3.7 \mathrm{MW} \mathrm{ICRH}$ off-axis, 9.1 MW NBI. The reference temperature is indicated in the plot, the lines are the borders between edge, stiff and core regions (compare with figure 9). As expected, the pulse with on-axis heating has a wider stiff region.

Profiles obtained in JET plasmas when combining mode conversion ICRF and NBI heating are shown on figure 10 [21]. The scaling temperature $T_{\mathrm{gB}}$ is indicated on each profile. A change of slope occurs in general when $T_{\mathrm{e}} \approx T_{\mathrm{gB}}$, except for plasmas in ASDEX-Upgrade at $I_{\mathrm{p}}=1 \mathrm{MA}$, where equation (8) predicts a value of $T_{\mathrm{gB}}$ that is too large. Interestingly, using a value of $T_{\mathrm{gB}}$ calculated using the values of $\chi_{\mathrm{s}}$ and $\kappa_{\mathrm{c}}$ deduced from the two-term scaling law (see next section, equation (29)) is in better agreement with experiment. We note also that the change of slope is sharper than expected (the transition at $T_{\mathrm{e}} \approx T_{\mathrm{gB}}$ is a smooth one). At this point, it is stressed that other reasons may explain the different behaviour in the edge: for instance a change of the underlying instability, the influence of the scrape-off layer or an effect of collisionality.

Another interesting feature is the change of gradients that occurs in JET core plasmas. No sawtooth is observed in the plasmas shown in figure 10. So this change of slope may correspond to the transition between stiff and core regions that is expected when the gradient length crosses the stability threshold. Note that the ASDEX-Upgrade plasmas at $I_{\mathrm{p}}=1 \mathrm{MA}$ shown in figure 9 (right panel) exhibit sawteeth, which flatten the temperature profile. Thus the change of slope at $\rho=0.45$ probably corresponds to the $q=1$ magnetic surface, masking a possible transition from stiff to non-stiff regions. The transition from the stiff region to the edge region is believed to be at $\rho \approx 0.8$ for the Ohmic case and at $\rho \approx 0.9$ for the case $P_{\mathrm{ECRH}}=1.6 \mathrm{MW}$. This is in agreement with the model prediction that the stiff region extends further out with increasing temperature.

Another analysis consists of drawing $-R \nabla T_{\mathrm{e}} / T_{\mathrm{e}}$ versus $T_{\mathrm{e}} / T_{\mathrm{gB}}$. For a stiff profile, this curve is expected to be universal at a given value of $\lambda_{0, \mathrm{e}}$, as shown by equation (10). The exercise has been done in ASDEX-Upgrade (figure 11) and in JET (figure 12). These figures exhibit some similarity with figure 1 . The main features predicted by the model equation (1) are recovered. In particular, the region where the gradient length is close to the threshold is wider when the heat deposition profile is more localized in the core. This trend is observed when comparing ECRH with Ohmic heating (figure 11), or when comparing off-axis with on-axis heating in JET plasmas (see figure 12). 


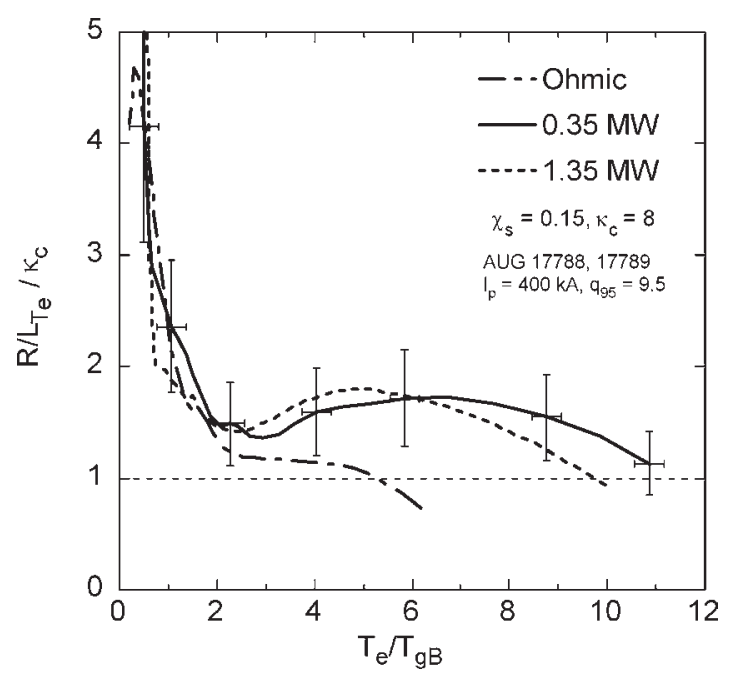

Figure 11. $-R \nabla \log T_{\mathrm{e}} / \kappa_{\mathrm{c}}$ versus $T_{\mathrm{e}} / T_{\mathrm{gB}}$ in ASDEX-Upgrade.

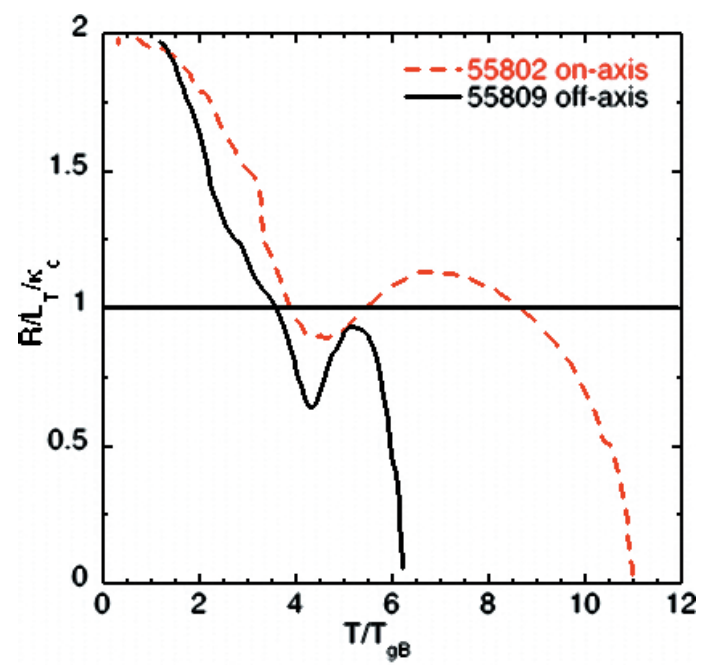

Figure 12. $-R \nabla \log T_{\mathrm{e}} / \kappa_{\mathrm{c}}$ versus $T_{\mathrm{e}} / T_{\mathrm{gB}}$ in JET.

\subsection{Comparison with a two term scaling law}

The ITPA group has proposed two scaling laws for the energy content of the plasma bulk [8]. They correspond to two different hypotheses for the physics underlying the confinement in the pedestal region. The first one assumes that the edge confinement is controlled by thermal conduction with some degradation with $\beta$, while the second relies on an MHD $\beta$ limit within the pedestal region. In terms of accuracy, these two models are equivalent. We will analyse in detail the model with MHD limited edge, because it corresponds to a confinement in the bulk that is gyroBohm and electrostatic, i.e. consistent with the assumptions underlying the model used here. More precisely the scaling law for the bulk region is

$$
W_{\text {ITPA, bulk }}=0.15 M^{0.34} \kappa^{-0.34} \varepsilon_{\mathrm{a}}^{1.96} R^{2.32} I_{\mathrm{p}, \mathrm{MA}}^{0.68} B_{\mathrm{T}}^{0.13} n_{\mathrm{a}, 19}^{0.59} P_{\mathrm{MW}}^{0.42} \text {. }
$$




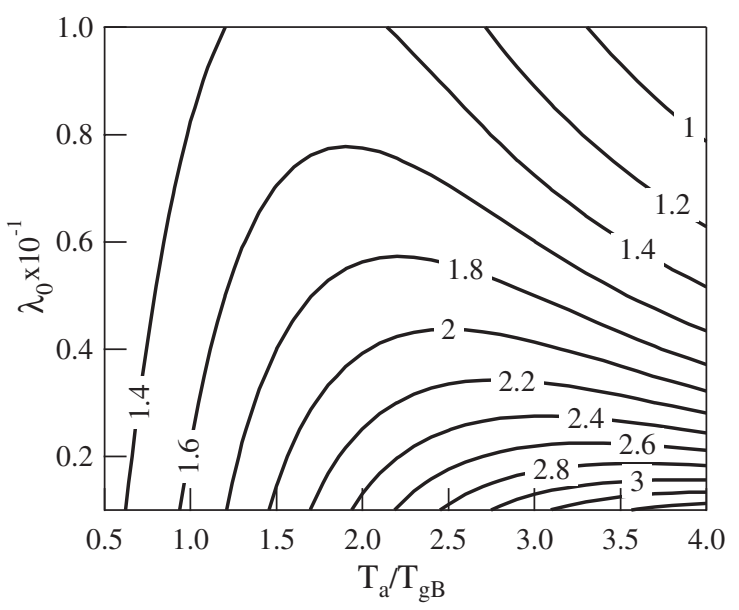

Figure 13. Contour lines of the form factor $C_{\text {ITPA }}$ versus the normalized edge temperature and degree of stiffness $\lambda_{0}$.

The corresponding normalized confinement time $B \tau_{E}$ scales as $\rho_{*}^{-3} \beta^{0.05}$, where $\rho_{*}$ is the normalized gyroradius and $\beta$ is the ratio of plasma kinetic pressure to magnetic pressure. This scaling is close to an electrostatic gyroBohm scaling law. Using the relation

$$
q_{\mathrm{a}}=5 \frac{1+\kappa^{2}}{2} \frac{a^{2} B}{R I_{\mathrm{p}, \mathrm{MA}}},
$$

one finds

$W_{\text {ITPA, bulk }}=0.45 M^{0.34} \kappa^{-0.34}\left(\frac{1+\kappa^{2}}{2}\right)^{0.68} \varepsilon_{\mathrm{a}}^{3.32} R^{3} q_{\mathrm{a}}^{-0.68} B_{\mathrm{T}}^{0.81} n_{\mathrm{a}, 19}^{0.59} P_{\mathrm{MW}}^{0.42}$

to be compared with the equation (19) when using the expression of $\tau_{\mathrm{gB}}$ given by equation (A.11)

$W_{\text {bulk }}=0.179 C_{\text {ITPA }} \chi_{\mathrm{s}, \text { eff }}^{-2 / 5} \kappa_{\mathrm{c}}^{-4 / 5} M^{-1 / 5} \kappa^{7 / 5}\left(\frac{1+\kappa^{2}}{2}\right)^{-2 / 5} \varepsilon_{\mathrm{a}}^{8 / 5} R^{3} q_{\mathrm{a}}^{-2 v / 5} B_{\mathrm{T}}^{4 / 5} n_{\mathrm{a}, 19}^{3 / 5} P_{\mathrm{MW}}^{2 / 5}$.

The exponents of power, density, magnetic field, safety factor and major radius are similar in equations (27) and (28). Note that the agreement for the safety factor comes from the choice $v=\frac{3}{2}$. Some significant differences between equations (27) and (28) appear in the exponent of the inverse aspect ratio $\varepsilon_{\mathrm{a}}=a / R$, elongation and mass, thus suggesting a dependence of the stiffness parameter on those quantities. Part of the difficulty comes from the dependence on elongation that is not a power law. One way to make an estimate is to assume that $\kappa$ is close to 1 , so that $\left(1+\kappa^{2}\right) / 2$ behaves as $\kappa$. Putting all differences in the stiffness factor, it is found that the two scaling laws agree if $\chi_{\mathrm{s} \text {, eff }}$ behaves as $\kappa^{1.7} M^{-1.3} \varepsilon_{\mathrm{a}}^{-4.3}$. While the variations with elongation and mass number may be possibly reconciled with physics considerations, it is difficulty to justify such a strong variation with the inverse aspect ratio. Assuming a 'typical' case $\kappa=1.6, \varepsilon_{\mathrm{a}}=\frac{1}{3}$ and $M=2$, it is found that equation (27) matches equation (28) if $\chi_{\mathrm{s}, \text { eff }}^{2 / 5} \kappa_{\mathrm{c}}^{4 / 5}=2.20 C_{\text {ITPA }}$. The contour lines of $C_{\text {ITPA }}$ are shown in figure 13 for a large domain of variation of the parameters $\lambda_{0}$ and $T_{\mathrm{a}} / T_{\mathrm{gB}}$. It is found that $C_{\text {ITPA }}$ remains in the interval $1-3$. Choosing $\kappa_{\mathrm{c}} \approx 5$, one finds that $\chi_{\mathrm{s} \text {, eff }}$ should be in the range 0.3-4.5. Choosing a medium value $C_{\mathrm{ITPA}}=2.0$ and $\kappa_{\mathrm{c}} \approx 5$ yields a stiffness factor $\chi_{\mathrm{s} \text {,eff }} \approx 1.6$. It is reminded here that $\chi_{\mathrm{s} \text {, eff }}$ is an effective stiffness factor. For equal temperatures, $\chi_{\mathrm{s} \text {, eff }}$ is the 


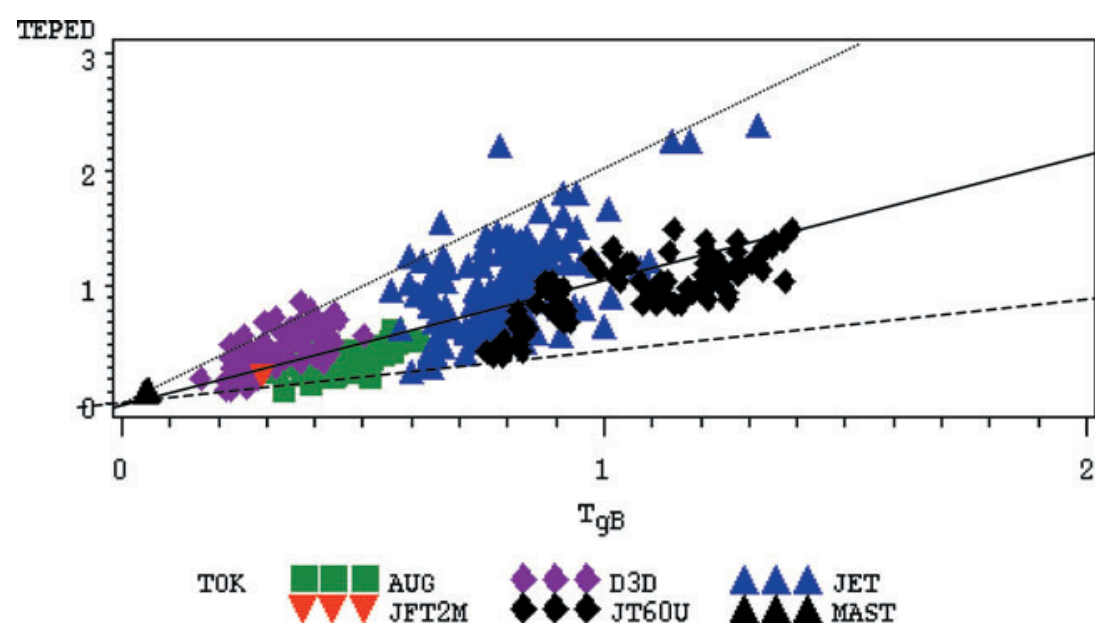

Figure 14. Pedestal temperature $T_{\mathrm{a}}$ versus the scaling temperature $T_{\mathrm{gB}}$. The lines indicate the identities $\left.T_{\mathrm{a}}=0.5 T_{\mathrm{gB}}(--)\right), T_{\mathrm{a}}=T_{\mathrm{gB}}(-)$ and $T_{\mathrm{a}}=2 T_{\mathrm{gB}}(\cdots \cdots)$.

sum of electron and ion stiffness parameters, $\chi_{\mathrm{s}, \mathrm{eff}}=\chi_{\mathrm{s}, \mathrm{e}}+\chi_{\mathrm{s}, \mathrm{i}}$. Thus $\chi_{\mathrm{s}, \mathrm{e}}$ and $\chi_{\mathrm{s}, \mathrm{i}}$ should be of order unity. The choice $\chi_{\mathrm{s} \text {, eff }} \approx 1.6$ leads to the following 'practical' expressions of the scaling temperature $T_{\mathrm{gB}}$ and the energy content of the plasma bulk.

$$
T_{\mathrm{gB}, \mathrm{keV}} \approx 0.43\left(\frac{1+\kappa^{2}}{2 \kappa}\right)^{-2 / 5} M^{-1 / 5} \varepsilon_{\mathrm{a}}^{-2 / 5} q_{\mathrm{a}}^{-3 / 5} B_{\mathrm{T}}^{4 / 5} n_{\mathrm{a}, 19}^{-2 / 5} P_{\mathrm{MW}}^{2 / 5}
$$

and

$$
W_{\text {bulk }} \approx 0.081 M^{-1 / 5} \kappa^{7 / 5}\left(\frac{1+\kappa^{2}}{2}\right)^{-2 / 5} \varepsilon_{\mathrm{a}}^{8 / 5} R^{3} q_{\mathrm{a}}^{-3 / 5} B_{\mathrm{T}}^{4 / 5} n_{\mathrm{a}, 19}^{3 / 5} P_{\mathrm{MW}}^{2 / 5} .
$$

The ITPA database has been used to compare the pedestal height $T_{\mathrm{a}}$ to $T_{\mathrm{gB}}$, given by the expression (29). The result is shown in figure 14. It is found that $T_{\mathrm{a}}$ ranges between $0.5 T_{\mathrm{gB}}$ and $2 T_{\mathrm{gB}}$. However this result must be considered with caution since the stiffness factor could depart significantly from the value $\chi_{\mathrm{s}, \text { eff }} \approx 1.6$ chosen here. The trend is that the ratio $T_{\mathrm{a}} / T_{\mathrm{gB}}$ is greater in large devices JT-60U and JET than in the medium size devices DIII-D and ASDEXUpgrade. This is an interesting feature in view of figure 3 . Indeed, the stiff region was found to reach its maximum size for $T_{\mathrm{a}} \approx T_{\mathrm{gB}}$ and decreases when $T_{\mathrm{a}} / T_{\mathrm{gB}}$ increases. This may explain why large devices are claimed to be less stiff (or not stiff at all). In spite of a higher pedestal that makes the edge stiffer, the width of the core region, which is sub-critical, could be larger in those plasmas, in particular in the H-mode.

A pending question is the compatibility of the two-term scaling law with the values of stiffness factor deduced from modulation experiments (table 1). This is a delicate point since most of these plasmas are in L-mode, with ratios $T_{\mathrm{e}} / T_{\mathrm{i}}$ different from 1 , whereas the ITPA database deals with H-mode plasmas, with $T_{\mathrm{e}} \approx T_{\mathrm{i}}$. It is expected that the core physics is the same in L- and H-modes. Similar values of $\lambda_{\mathrm{s}, \mathrm{e}}$ were found in the L-mode (\#55809) and H-mode (\#58148) plasmas on JET, thus providing some support to this assumption. The range of stiffness factor $\chi_{\mathrm{s} \text {,eff }}$ that is compatible with the global scaling law is $0.3-4.5$. It is reminded here that $\chi_{\mathrm{s}, \text { eff }}=\left(T_{\mathrm{e}} / T_{\mathrm{i}}\right)^{5 / 2} \chi_{\mathrm{s}, \mathrm{e}}+\chi_{\mathrm{s}, \mathrm{i}}$. Regarding the values of stiffness factor obtained from heat wave analysis, the highest values $\chi_{\mathrm{s}, \mathrm{e}}=3-6$ obtained at JET (and for one pulse of ASDEX-Upgrade) appear too large by a factor 1.5-2.5, assuming $\chi_{\mathrm{s}, \mathrm{e}}=\chi_{\mathrm{s}, \mathrm{i}}$. A sensitivity 
study indicates that the heat wave analysis becomes less sensitive to the value of $\chi_{\mathrm{s}, \mathrm{e}}$ at high $\chi_{\mathrm{s}, \mathrm{e}}$ values. Moreover it can be noted that a significant scatter of points is present in figure 8 , which is mainly due to the fact that the simple model assumed here does not always allow a perfect reproduction of the data, and some trade off in the fit of the various quantities often needs to be accepted. Taking also into account the various simplifications introduced into this analysis, we consider that these results are encouraging.

Finally, it is interesting to compare the present range of stiffness factors with theoretical expectations. The IFS-PPPL model [12] is close to the form of equation (1) when simplified, i.e. choosing $Z_{\text {eff }}=1, r / R \ll 1, R / L_{\mathrm{n}}=0$,

$$
\chi_{T_{\mathrm{i}}}=\frac{12}{1+s^{0.84}}\left(q \frac{T_{\mathrm{e}}}{T_{\mathrm{i}}}\right)^{1.1} \frac{c_{\mathrm{s}} \rho_{\mathrm{s}}^{2}}{R}\left(\frac{-R \partial_{r} T}{T}-\kappa_{\mathrm{c}}\right) H\left(\frac{-R \partial_{r} T}{T}-\kappa_{\mathrm{c}}\right) .
$$

We note that the exponent of $q$ is $v=1.1$. Choosing $T_{\mathrm{i}}=T_{\mathrm{e}}$, and a magnetic shear $s=1$ one gets $\chi_{\mathrm{s}, \mathrm{i}} \approx 6$. An estimate of $\chi_{\mathrm{s}, \mathrm{e}}$ is harder to provide, since it depends on collisionality.

The GLF23 model [13] is a more sophisticated model, which uses a calculation of growth rates. So it cannot be easily reduced to an expression of the form of equation (1). However it was mentioned in the original paper that the stiffness factor is similar to the IFS-PPPL model. A renormalized model was proposed recently [32] where the stiffness factor of ITG/TEM driven transport was reduced by a factor 3.7. Using the IFS-PPPL value, this leads to an estimate $\chi_{\mathrm{s}, \mathrm{i}} \approx 1.6$. The Weiland model is also based on a comprehensive calculation of growth rates. Numerical scans indicate that $\chi_{\mathrm{s}, \mathrm{i}} \approx 1$ and $\chi_{\mathrm{s}, \mathrm{e}} \approx 0.3$. Finally several theoretical models and turbulence simulations were compared in the CYCLONE group [22]. The results from the LLNL gyrokinetic code were fitted by the expression

$$
\chi_{T_{\mathrm{i}}}=5 \frac{c_{\mathrm{s}} \rho_{\mathrm{s}}^{2}}{R}\left(\frac{-R \partial_{r} T}{T}-\kappa_{\mathrm{c}}\right) H\left(\frac{-R \partial_{r} T}{T}-\kappa_{\mathrm{c}}\right),
$$

where $\kappa_{\mathrm{c}}=6$. The simulations were done at fixed ratio of density to temperature gradient length $L_{\mathrm{n}} / L_{\mathrm{T}}=3.1$, with $Z_{\text {eff }}=1$. We note that there is no $q$ dependence in this expression, i.e. $\chi_{\mathrm{s}, \mathrm{i}}=5 / q^{v}$. Since the simulations were done at $q=1.4$, one gets an estimate $\chi_{\mathrm{s}, \mathrm{i}} \approx 3$. In summary, it is found that $\chi_{\mathrm{s}, \mathrm{i}}$ ranges between 1 and 6 depending on the model. The electron stiffness factor is less documented. It is typically three times smaller that the ion stiffness. We stress here that these values are purely indicative, as these models exhibit rather complex parametric dependences. Nevertheless, we can conclude that the range of variation of the stiffness factor found in the literature is quite large, and compatible with both our experimental results and the value deduced from the ITPA scaling law.

\section{Summary}

Using the critical gradient transport model

$$
\chi_{T}=\chi_{\mathrm{s}} q^{\nu} \frac{T}{e B} \frac{\rho_{\mathrm{s}}}{R}\left(\frac{-R \partial_{r} T}{T}-\kappa_{\mathrm{c}}\right) H\left(\frac{-R \partial_{r} T}{T}-\kappa_{\mathrm{c}}\right)+\chi_{0} q^{\nu} \frac{T}{e B} \frac{\rho_{\mathrm{s}}}{R},
$$

it is found that:

(1) Choosing an exponent $v=\frac{3}{2}$, the proximity of a profile to a marginally stable state (stiffness) is then characterized by two numbers for each species for a given heat deposition profile: the degree of stiffness $\lambda_{0}=\chi_{0} / \kappa_{\mathrm{c}} \chi_{\mathrm{s}}$, and the normalized edge temperature $T_{\mathrm{a}} / T_{\mathrm{gB}}$. Here $T_{\mathrm{a}}$ is the edge temperature and the reference temperature $T_{\mathrm{gB}}$ satisfies the condition

$$
\Gamma_{T}(a)=\chi_{\mathrm{s}} \kappa_{\mathrm{c}}^{2} q_{\mathrm{a}}^{v} n_{\mathrm{a}} T_{\mathrm{gB}} \frac{T_{\mathrm{gB}}}{e B} \frac{\rho_{\mathrm{s} 0}}{R^{2}}
$$


where $\Gamma_{T}(a)$ is the thermal flux at the edge. The plasma is characterized by three regions: a core region that is subcritical, an edge region where the temperature logarithmic gradient is above the threshold, and a stiff region where the profile is close to marginal stability. The respective sizes of these three regions depend on the degree of stiffness $\lambda_{0}$ and the normalized edge temperature $T_{\mathrm{a}} / T_{\mathrm{gB}}$. The width of the central (stiff) region becomes wider when $\lambda_{0}$ decreases. When the normalized edge temperature $T_{\mathrm{a}} / T_{\mathrm{gB}}$ increases, the edge region shrinks and ultimately disappears. However the core (subcritical) region becomes wider, leading to a smaller intermediate stiff region (figure 3 ).

(2) The energy content of the plasma bulk (without the pedestal contribution and for an elliptical geometry) is

$W_{\text {bulk }}=0.179 C_{\text {ITPA }} \chi_{\mathrm{s}, \text { eff }}^{-2 / 5} \kappa_{\mathrm{c}}^{-4 / 5} M^{-1 / 5} \kappa^{7 / 5}\left(\frac{1+\kappa^{2}}{2}\right)^{-2 / 5} \varepsilon_{\mathrm{a}}^{8 / 5} R^{3} B_{\mathrm{T}}^{4 / 5} q_{\mathrm{a}}^{-3 / 5} n_{\mathrm{a}, 19}^{3 / 5} P_{\mathrm{MW}}^{2 / 5}$.

In this formula, the stiffness factor $\chi_{\mathrm{s}, \text { eff }}$ is an effective value $\chi_{\mathrm{s}, \mathrm{eff}}=\chi_{\mathrm{s}, \mathrm{e}}+\chi_{\mathrm{s}, \mathrm{i}}$, where $\chi_{\mathrm{s}, \mathrm{e}}$ and $\chi_{\mathrm{s}, \mathrm{i}}$ are the electron and ion stiffness factors (the threshold $\kappa_{\mathrm{c}}$ and the degree of stiffness $\lambda_{0}$ are supposed to be the same for electrons and ions). The form factor $C_{\text {ITPA }}$ depends on the normalized edge temperature $T_{\mathrm{a}} / T_{\mathrm{gB}}$ and the degree of stiffness $\lambda_{0}$. It decreases with the normalized edge temperature for non-stiff profiles. On the contrary it increases linearly with the edge temperature for stiff profiles. In the general case, $C_{\text {ITPA }}$ is a sum of the contributions of stiff and non-stiff regions, which behave in opposite ways. The two-term scaling law proposed by the ITPA-CDBM Group corresponds to intermediate values of $\lambda_{0}$, typically between 0.01 and 0.1 , which indeed is approximately the range of values observed in the heat modulation experiments for electrons (table 1). Choosing this range of stiffness factors and varying the edge temperature in a wide domain, it is found that $1<C_{\text {ITPA }}<3$.

(3) This model has been applied for electrons at JET, ASDEX-Upgrade, TORE SUPRA and FTU (mainly modulation experiments in the electron channel). The threshold was found to lie in the expected domain for ITG/TEM modes. On the other hand, the stiffness parameter $\chi_{\mathrm{s}, \mathrm{e}}$ was found to cover a wide range of variation, i.e. it ranges between 0.15 and 6 .

(4) A comparison between this transport model and the ITPA two-term scaling law leads to the relation $\chi_{\mathrm{s}, \text { eff }}^{2 / 5} \kappa_{\mathrm{c}}^{4 / 5}=2.2 C_{\text {ITPA }}$. Choosing $C_{\text {ITPA }}$ in the range $1-3$ and $\kappa_{\mathrm{c}} \approx 5$, one finds that $\chi_{\mathrm{s} \text {, eff }}$ should be in the range $0.3-4.5$. The medium value $C_{\text {ITPA }}=2.0$ yields a stiffness parameter $\chi_{\mathrm{s} \text {,eff }} \approx 1.6$, i.e. a value of the order of unity for each species. The range $\chi_{\mathrm{s}, \text { eff }} \approx 0.3-4.5$ is compatible with the results found in JET, ASDEXUpgrade, TORE SUPRA and FTU $\left(\chi_{\mathrm{s}, \mathrm{e}} \approx 0.15-6\right)$, given the various sources of uncertainty. Also it is compatible with theoretical predictions. A brief survey of the literature yields an ion stiffness number $\chi_{\mathrm{s}, \mathrm{i}}$ that ranges typically between 1 and 6 . Although electron stiffness is less documented, the stiffness number $\chi_{\mathrm{s}, \mathrm{e}}$ ranges typically between 0.3 and 2 $\left(\chi_{\mathrm{s}, \mathrm{e}} \approx 0.3-2\right)$.

\section{Conclusion}

A critical gradient transport model that covers the basic properties of turbulent transport has been used extensively. This model is parameterized by three scalars for each species which have to be deduced from experiment. It also provides a quantitative criterion to get stiff profiles, thus providing a way to assess quantitatively this controversial issue. The plasma covers three regions: a core region that is subcritical, an edge region where the temperature logarithmic gradient is above the threshold and a stiff region in between, where the profile is close to marginal stability. The respective sizes of these three regions depend on the degree of stiffness and the edge temperature normalized in an appropriate way. When the normalized 
edge temperature increases, the edge region shrinks and ultimately disappears. However the core (subcritical) region becomes wider, leading ultimately to a favourable situation where the radial extent of the stiff region gets smaller. The existence of three regions in the plasma, one only being stiff, explains why a transport model with a threshold can still be compatible with the two term scaling law developed by the ITPA-CDBM Group. This model has been applied to analyse a variety of experiments using mostly electron heat modulation on JET, ASDEX-Upgrade, TORE SUPRA and FTU. The thresholds are found to be in the expected domain for ITG/TEM modes. However, it has been found that the range of variation of the stiffness parameter is very large. This wide range is compatible with the broad ensemble of values predicted by various theories. On the other hand, it is larger than the domain of variation estimated from the ITPA-CDBM two term scaling law. This difference appears to be compatible with the simplifications underlying this transport model, and also with the various uncertainties that are encountered in determining the model's parameters from modulation experiments and global scaling laws.

Thus these results can be considered as encouraging. Still further experiments are needed to better determine the parametric dependences of the threshold and, more importantly, of the stiffness factor. This study suggests a dependence on the logarithmic gradient of the ion temperature. This raises the question of the coupling between electron and ion channels, or of the stability between ITG and TEM branches. This coupling may lead to a dependence of the heat flux of one species on the temperature gradient length of another species, in addition to the temperature itself. The clarification of this point will require further experiments in a variety of electron and ion heating scenarios. Also the value of the stiffness factor depends sensitively on the choice of scaling that is done with respect to the safety factor and geometrical factors. The latter point is illustrated by the difference between the two term global scaling law and the present model in terms of shaping and aspect ratio parameters. Regarding this question, changing the profile of the safety factor would be useful, for instance by generating a non-inductive part of the current. The last issue is the nature and scaling of the background diffusivity that plays an important role, because it determines the width of the non-stiff core region. Reducing the uncertainty in this parameter would allow a more reliable comparison. Also the present model should be used more extensively in H-mode plasmas, to be compared directly with the ITPA database.

\section{Appendix A. Normalization of the heat equation}

The heat flux is linked to the gradient by the Fourier Law

$$
\Gamma_{T}=-n \chi_{T} \partial_{r} T
$$

It is also the radial integral of the heat source $P_{\text {heat }}$

$$
\Gamma_{T}=\frac{1}{\left\langle|\nabla r|^{2}\right\rangle V^{\prime}} \int_{0}^{r} \mathrm{~d} r V^{\prime} P_{\text {heat }}(r)
$$

where

$$
V^{\prime}=2 \pi \psi^{\prime} \int_{0}^{2 \pi} \frac{\mathrm{d} \theta}{\boldsymbol{B} \cdot \nabla \theta} .
$$

We now solve the heat equation (A.2) with the transport model, equation (1). It is convenient to introduce a normalized spatial coordinate

$$
\rho=\frac{5}{2} \kappa_{\mathrm{c}} \frac{a-r}{R}
$$


(the magnetic axis is located at $\rho=\rho_{\max }=5 \kappa_{\mathrm{c}} a / 2 R$ and the edge at $\rho=0$, also $\left.\rho / \rho_{\max }=1-r / a\right)$. The parameter

$$
\lambda_{0}=\frac{\chi_{0}}{\kappa_{\mathrm{c}} \chi_{\mathrm{s}}}
$$

characterizes the relative degree of stiffness and is supposed to be smaller than 1 . Finally we define a normalized temperature

$$
\tau=\left(\frac{T}{T_{\mathrm{gB}}}\right)^{5 / 2}
$$

and a normalized heat flux $g(\rho)=G(r) / G(a)$ with

$$
G(r)=\frac{1}{n(r)[q(r)]^{\nu}\left\langle|\nabla r|^{2}\right\rangle V^{\prime}(r)} \int_{0}^{r} \mathrm{~d} r V^{\prime}(r) P_{\text {heat }}(r) .
$$

The relation

$$
\Gamma_{T}(a)=\chi_{\mathrm{s}} \kappa_{\mathrm{c}}^{2} q_{\mathrm{a}}^{\nu} n_{\mathrm{a}} T_{\mathrm{gB}} \frac{T_{\mathrm{gB}}}{e B} \frac{\rho_{\mathrm{s}, \mathrm{gB}}}{R^{2}}
$$

defines the temperature $T_{\mathrm{gB}}$. The flux $\Gamma_{T}(a)$ is equal to the heating power $P$ divided by a surface $S=C_{\mathrm{s}} 2 \pi R 2 \pi a ; q_{\mathrm{a}}$ is the edge safety factor and $n_{\mathrm{a}}$ the edge density. The shape factor $C_{\mathrm{s}}$ is defined as

$$
C_{\mathrm{s}}=\frac{\left\langle|\nabla r|^{2}\right\rangle_{r=a} V_{\mathrm{a}}^{\prime}}{2 \pi R 2 \pi a} .
$$

For an elliptical geometry, $\left\langle|\nabla r|^{2}\right\rangle=\left(1+\kappa^{2}\right) / 2 \kappa^{2}, V^{\prime}=2 \pi R 2 \pi \kappa r$ and $C_{\mathrm{s}}=\left(1+\kappa^{2}\right) / 2 \kappa$, where $\kappa$ is the elongation.

The temperature $T_{\mathrm{gB}}$ exhibits the usual gyroBohm scaling. It will play an important role in this calculation. It may be recast in a more convenient way when the geometry is elliptical

$T_{\mathrm{gB}, \mathrm{keV}}=1.89\left(\frac{1+\kappa^{2}}{2 \kappa}\right)^{-2 / 5} \chi_{\mathrm{s}}^{-2 / 5} \kappa_{\mathrm{c}}^{-4 / 5} M^{-1 / 5} \varepsilon_{\mathrm{a}}^{-2 / 5} q_{\mathrm{a}}^{-2 v / 5} B_{\mathrm{T}}^{4 / 5} n_{\mathrm{a}, 19}^{-2 / 5} P_{\mathrm{MW}}^{2 / 5}$.

We also define a reference gyroBohm energy content $W_{\mathrm{gB}}$

$$
W_{\mathrm{gB}}(\mathrm{MJ})=\frac{3 n_{\mathrm{a}} T_{\mathrm{gB}} V}{P}=0.179 C_{\mathrm{sh}} \chi_{\mathrm{s}}^{-2 / 5} \kappa_{\mathrm{c}}^{-4 / 5} M^{-1 / 5} R^{3} \varepsilon_{\mathrm{a}}^{8 / 5} q_{\mathrm{a}}^{-2 v / 5} B_{\mathrm{T}}^{4 / 5} n_{\mathrm{a}, 19}^{3 / 5} P_{\mathrm{MW}}^{2 / 5} .
$$

The shaping factor $C_{\mathrm{sh}}$ is $C_{\mathrm{sh}}=C_{\mathrm{v}} C_{\mathrm{s}}^{-2 / 5}$ where the volume form factor $C_{\mathrm{v}}$ is such that $V=C_{\mathrm{v}} 2 \pi R \pi a^{2}$. In elliptical geometry $C_{\mathrm{s}}=\left(1+\kappa^{2}\right) / 2 \kappa$ and $C_{\mathrm{v}}=\kappa$, so that $C_{\text {sh }}=\kappa^{7 / 5}\left(\left(1+\kappa^{2}\right) / 2\right)^{-2 / 5}$. The units are: length in metre, $B_{\mathrm{T}}$ in Tesla, density in $10^{19} \mathrm{~m}^{-3}$ and power in MW. $\varepsilon_{\mathrm{a}}=a / R$ is the inverse aspect ratio. As an illustration, the values $\kappa_{\mathrm{c}}=5$ and $\varepsilon_{\mathrm{a}}=\frac{1}{3}$ (i.e. $\rho_{\max }=5$ ) will be chosen in the following. Typical values are $\chi_{0} \approx 0.1$, $\chi_{\mathrm{s}} \approx 1$ and $\kappa_{\mathrm{c}}=4-6$. The normalized ratio $\lambda_{0}=\chi_{0} / \kappa_{\mathrm{c}} \chi_{\mathrm{s}}$ is therefore a small number. The value $\lambda_{0}=0.025$ is chosen as an example in this paper. With the above normalization, the heat law becomes particularly simple $\left(\tau^{\prime}=\partial_{\rho} \tau\right)$

$$
\tau^{\prime}\left(\frac{\tau^{\prime}}{\tau}-1\right) H\left(\frac{\tau^{\prime}}{\tau}-1\right)+\lambda_{0} \tau^{\prime}=g
$$

\section{Appendix B. Analytic solution for constant or exponential flux}

If the heat flux $g(\rho)$ is a constant $(=1)$, the solution of equation $(11)$ is analytic, i.e. using the change of variable $\cosh (u)=\left(1-\lambda_{0}\right)^{2}(\tau / 2)+1$. 
Above the threshold. $\quad 0<\rho<\rho_{\mathrm{cr}}, \tau_{\mathrm{a}}<\tau<1 / \lambda_{0}$

$\rho=F(\tau)-F\left(\tau_{\mathrm{a}}\right)$,

$$
\begin{aligned}
& F(\tau)=\frac{1}{1-\lambda_{0}} \log \left\{1+\left(1-\lambda_{0}\right)^{2} \frac{\tau}{2}+\left(1-\lambda_{0}\right)\left[\left(1-\lambda_{0}\right)^{2} \frac{\tau^{2}}{4}+\tau\right]^{1 / 2}\right\} \\
& +\left[\left(1-\lambda_{0}\right)^{2} \frac{\tau^{2}}{4}+\tau\right]^{1 / 2}-\left(1-\lambda_{0}\right) \frac{\tau}{2}, \\
& \rho_{\mathrm{cr}}=-\frac{1}{1-\lambda_{0}} \log \left(\lambda_{0}\right)+1-F\left(\tau_{\mathrm{a}}\right), \\
& \rho_{\mathrm{gB}}=F(1)-F\left(\tau_{\mathrm{a}}\right) .
\end{aligned}
$$

Approximate expressions of the function $F$ are the following

$$
\tau \gg 1, \quad \frac{F(\tau) \approx \log (\tau)}{1-\lambda_{0}} ; \quad \tau \ll 1, \quad F(\tau) \approx 2 \tau^{1 / 2} .
$$

Below the threshold. $\rho>\rho_{\mathrm{cr}}, \tau>1 / \lambda_{0}$

$$
\tau=\frac{1+\rho-\rho_{\mathrm{cr}}}{\lambda_{0}}
$$

If the heat source is exponential $g(\rho)=\exp \left[\left(1-\lambda_{0}\right) \rho\right]$, one may make the change of function

$$
\tau=\theta \mathrm{e}^{\left(1-\lambda_{0}\right) \rho} ; \quad \tau_{\mathrm{a}}=\theta_{\mathrm{a}} .
$$

Above the threshold, the function $\theta$ is then solution of the equation

$$
\theta^{\prime 2}+\theta\left(1-\lambda_{0}\right) \theta^{\prime}-g \theta=0 \text {. }
$$

This equation is close to equation (12). Its solution is

$$
\rho=G(\theta)-G\left(\theta_{\mathrm{a}}\right), \quad G(\theta)=F(\theta)+\left(1-\lambda_{0}\right) \theta .
$$

The transition between the stiff and core region occurs when $\theta=1 / \lambda_{0}$, i.e. at the position

$$
\rho_{\mathrm{cr}}=-\frac{1}{\left(1-\lambda_{0}\right)} \log \left(\lambda_{0}\right)+\frac{1}{\lambda_{0}}-G\left(\theta_{\mathrm{a}}\right) .
$$

Note that this value is much larger than in the constant $g$ case. This confirms that a more peaked heating source induces a stiffer profile. The solution in the core region is

$$
\tau=\frac{1}{1-\lambda_{0}}\left(\frac{1}{\lambda_{0}} g(\rho)-g\left(\rho_{\mathrm{cr}}\right)\right)
$$

\section{Appendix C. Asymptotic expressions of the edge, core and stiff region contributions to the confinement}

Edge region. The edge contribution, which corresponds to $\tau_{\mathrm{a}}<\tau<1$, can be approximated by the expression (assuming a small radial extend in the edge so that $g \approx 1$ and $J \approx 2$ )

$$
C_{\tau \text { edge }}=\frac{1}{\rho_{\max }} \int_{\tau_{\mathrm{a}}}^{1} \mathrm{~d} \tau \tau^{-1 / 10}=\frac{10}{9 \rho_{\max }}\left(1-\tau_{\mathrm{a}}{ }^{9 / 10}\right) .
$$

The expression (C.1) can be recast as

$$
C_{\text {ITPA }, \text { edge }} \approx \frac{10}{9 \rho_{\max }}\left\{1-\left(\frac{T_{\mathrm{a}}}{T_{\mathrm{gB}}}\right)^{9 / 4}-\frac{9}{5} \frac{T_{\mathrm{a}}}{T_{\mathrm{gB}}}+\frac{9}{5}\left(\frac{T_{\mathrm{a}}}{T_{\mathrm{gB}}}\right)^{1 / 2}\right\} .
$$

It is interesting to note that this expression depends sensitively on the edge temperature. The reason is that the transport model is a nonlinear function of the temperature, thus leading to some profile resilience, even if there is no threshold involved. 
Core region. An approximate expression can be found assuming $g \approx a / r$,

$$
C_{\text {ITPA }, \text { core }} \approx\left(\frac{r_{0}}{a}\right)^{2} \int_{0}^{\infty} \mathrm{d} u \mathrm{e}^{-u}\left\{\left[\tau_{0}+\frac{\rho_{\max }}{2 \lambda_{0}} u\right]^{2 / 5}-\tau_{\mathrm{a}}^{2 / 5}\right\} .
$$

When there exists a stiff region, one has $\tau_{\mathrm{cr}} \approx a /\left(r_{\mathrm{cr}} \lambda_{0}\right)$ and the pedestal contribution is negligible. The core contribution to the form factor is then an increasing function of the edge temperature.

Stiff region. In the general case, the profile is stiff within a layer. The contribution of a stiff layer $\left[\rho_{\mathrm{cr}}, \rho_{\mathrm{gB}}\right]$ (temperature $\left.\left[\tau_{\mathrm{cr}}, 1\right]\right)$ is

$C_{\tau \text { stiff }}=2\left(\frac{5}{2 \rho_{\max }}\right)^{2}\left\{\left[\frac{2}{5}\left(\rho_{\max }-\rho_{\mathrm{cr}}\right)+1\right] \tau_{\mathrm{cr}}^{2 / 5}-\left[\frac{2}{5}\left(\rho_{\max }-\rho_{\mathrm{gB}}\right)+1\right]\right\}$.

In presence of edge and core regions $\tau_{\mathrm{cr}}=g_{\mathrm{cr}} / \lambda_{0}$ where $g_{\mathrm{cr}}$ is the normalized heat flux at the transition to the core region. Thus, this contribution varies as the width $\rho_{\mathrm{cr}}-\rho_{\mathrm{gB}}$ of the stiff region. When the edge temperature increases, the edge non-stiff region disappears and this width increases. It then shrinks because the core region broadens.

\section{References}

[1] Coppi B and Sharky N 1981 Nucl. Fusion 211363

[2] Ryter F et al 2001 Phys. Rev. Lett. 862325

[3] Hoang G T et al 2001 Phys. Rev. Lett. 87125001

[4] Mantica P et al 2002 Plasma Phys. Control. Fusion 442185

[5] Baker D R et al 2001 Phys. Plasmas 84128

[6] Peeters A G 2002 Nucl. Fusion 421376

[7] Mikkelsen D R et al 2003 Nucl. Fusion 4330

[8] Cordey G et al 2003 Nucl. Fusion 43670

[9] Takizuka T 1998 Plasma Phys. Control. Fusion 40851

[10] Rebut P H, Lallia P P and Watkins M L 1989 Plasma Physics and Control Nuclear Fusion Research 1988: Proc. 12th Int. Conf. (Nice, 1988) vol 2 (Vienna: IAEA) p 191

[11] Nordman H, Weiland J and Jarmen A 1990 Nucl. Fusion 30983

[12] Kotschenreuther M, Dorland W, Beer M A and Hammett G W 1995 Phys. Plasmas 22381

[13] Waltz R E et al 1997 Phys. Plasmas 42482

[14] Zhu P, Bateman G, Kritz A H and Horton W 2000 Phys. Plasmas 72898

[15] Ottaviani M, Horton W and Erba M 1997 Plasma Phys. Control. Fusion 391461

[16] Erba M et al 1997 Plasma Phys. Control. Fusion 39261

[17] Parail V et al 1997 Nucl. Fusion 37481

[18] Tardini Get al 2002 Nucl. Fusion 42 L11

[19] Imbeaux F, Ryter F and Garbet X 2001 Plasma Phys. Control. Fusion 431503

[20] Ryter F et al 2003 Nucl. Fusion 431396

[21] Mantica P et al 2002 Transient heat transport studies in JET conventional and advanced tokamak plasmas Fusion Energy 2002: Proc. 19th IAEA Fusion Energy Conf. (Lyon, 2002) EX/P1-04 (Vienna: IAEA)

[22] Dimits A M et al 2000 Phys. Plasmas 7969

[23] Garbet X and Waltz R E 1996 Phys. Plasmas 31898

[24] Ottaviani M and Manfredi G 2001 Nucl. Fusion 41637

[25] Waltz R, Candy J M and Rosenbluth M N 2002 Phys. Plasmas 91938

[26] Lin Z et al 2002 Size scaling of turbulent transport in tokamak plasmas Fusion Energy 2002: 19th IAEA Fusion Energy Conf. (Lyon, 2002) TH/1-1 (Vienna: IAEA)

[27] Waltz R E, Staebler G M, Dorland W, Kotschenreuther M and Konings J A 1997 Phys. Plasmas 42482

[28] Ottaviani M private communication

[29] Petty C C, Kinsey J E and Luce T C 2004 Phys. Plasmas 111011

[30] Mantica P et al 2003 Heat wave propagation experiments and modelling at JET: L-mode, H-mode and ITBs Proc. 30th EPS Conf. on Controlled Fusion and Plasma Physics (St Petersburg, 2003)

[31] Wolf R C et al 2003 Plasma Phys. Control. Fusion 451757

[32] Kinsey J et al 2003 Fusion Sci. Technol. 44763 